\title{
A special focus on 2D formulations for contact problems using a covariant description
}

Alexander Konyukhov, Karl Schweizerhof Universität Karlsruhe, Institut für Mechanik

Institut für Mechanik

Kaiserstr. 12, Geb. 20.30

76128 Karlsruhe

Tel.: +49 (0) 721/ 608-2071

Fax: +49 (0) 721/ 608-7990

E-Mail: ifm@uni-karlsruhe.de

www.ifm.uni-karlsruhe.de 


\title{
A special focus on $2 \mathrm{D}$ formulations for contact problems using a covariant description
}

\author{
Alexander Konyukhov, Karl Schweizerhof
}

2005

\begin{abstract}
.
A fully covariant description, based on the consideration of contact conditions especially for the $2 \mathrm{D}$ case is proposed. The description is based on a reconsideration of contact kinematics and all necessary operations such as derivatives in a specially chosen curvilinear coordinate system based on a curved geometry in plane. In addition, details of the finite element implementation are presented for the simple linear contact element. Special cases, requiring the update of history variables as well as their careful transfer over the element boundaries are illustrated by numerical examples. With these procedures artificial jumps in the contact forces can be avoided.
\end{abstract}

Keywords. covariant description, contact problem, friction

\section{INTRODUCTION}

In the literature various contact descriptions for an effective finite element implementation are available, which can be basically characterized by the following: from 2D to 3D formulations, from non-frictional to frictional contact. Some major references are cited in the following: Wriggers et. al. [23] used an elasto-plastic analogy and the penalty regularization for 2D frictional problems restricted to piecewise linear contact elements. Parisch [14] considered non-frictional 3D contact and Parisch and Lübbing (1997) [15] revised the procedure for frictional contact within the penalty method for piecewise bilinear surface elements. Peric and Owen [16] used the penalty method for 3D frictional contact problems with small deformations. The main characteristics of the cited investigations are that the penalty functional as well as its linearization were considered in a global coordinate system and restricted to linear resp. bilinear surface elements. Laursen and Simo [9], however, formulated the penalty based contact conditions and the return mapping algorithm via convective surface coordinates, but the following linearization performed in the global coordinate system led to an artificial non-symmetry of the tangent matrix in the case of sticking. Wriggers [26] could overcome this artefact using the idea of mesh tying functionals. General overviews over contact conditions and contact algorithms which are nowadays used in practice, are covered by the books of Wriggers [26] 
and Laursen [10], while the theoretical aspects of the regularization methods in contact mechanics can be found in Kikuchi and Oden [5]. Beyond that the covariant description proposed in Konyukhov and Schweizerhof [6], [7] allows a unified description of contact problems within the penalty method independently of the surface discretization. The method contains rather complicated mathematical transformations in the local 3D coordinate system, however, finally leading to the consistent formulation of frictional contact. Some advantages of this approach were shown, e.g. the sticking matrix preserves necessarily its symmetry.

In the current contribution, we aim to present the development in a more simple comparative manner for both 2D and 3D formulations. We will show the unity of $2 \mathrm{D}$ and $3 \mathrm{D}$ formulations, where the $2 \mathrm{D}$ case can be derived, from one hand, as a simplified case of the particular 3D geometry of contact surfaces and, from the other hand, can be constructed separately based on the differential geometry of 2D plane curves. This consideration has additional advantages, e.g. the subdivision of the contact tangent matrices into the "main", the "rotational" part and the "curvature" part has a pure geometrical meaning. It is also possible to distinguish a-priori various cases, where some of the parts are necessary or can be omitted.

The article is organized as follows. We start with 2D kinematics based on a curved geometry in a plane. The main results concerning the $3 \mathrm{D}$ covariant description can then be presented without extensive involvement into mathematics. For further details of the 3D description we refer to [7]. Two-dimensional contact will also be considered separately in $2 \mathrm{D}$ as well as a reduction of the 3D developments. In addition, we will compare to known formulations and present some numerical examples. A particular focus is on problems concerning contact points traversing edges of contact segments and on problems with reversible loading.

\section{Geometry and Kinematics of Contact}

Considering a special contact case - contact between two cylindrical infinite bodies with plane strain deformations, see Fig. 1, leads to a definition of a 2D contact. In this case a generatrix $\mathbf{G H}$ of the first cylindrical body is a contact line and corresponds to a contact line $\mathbf{G}^{\prime} \mathbf{H}$ ' which is also a generatrix but of the second cylindrical body. Thus, 3D contact which can be seen as an interaction between two surfaces is reduced to an interaction between two boundary curves in the 2D case, see Fig. 2. One of boundary curves is chosen as the master curve. A coordinate system is considered on the boundary, either for a surface in 3D or 
for a curve in 2D. Thus contact occurs or two bodies are coming into contact, if a slave point belonging to the second body $S$ penetrates into the master body, where penetration is defined as the shortest distance between the surfaces of the two bodies. For simplicity we assume now that the parameterization of the boundaries is sufficiently smooth.

\subsection{Nomenclature of the used symbols}

Throughout the article a tensor notation with regard to both, surface and curve geometry, are used, therefore, a short notation used in the contribution is provided:

$\xi$ - arbitrary parameterization of a curve, convective coordinate.

$\zeta$ - the normal coordinate for $2 \mathrm{D}$ bodies, if the description is based on a cylindrical geometry. The value $\zeta$ describes the penetration.

$s$ - length parameterization of a curve.

$\mathbf{r}_{s}$ - position vector of the master point.

$\boldsymbol{\rho}(\xi)$ - position vector of the projection point.

$\boldsymbol{\rho}_{\xi}, \boldsymbol{\rho}_{\xi \xi}$ - the first resp. second derivative of the position vector in the case of an arbitrary parameterization.

$\boldsymbol{\tau}$ - tangent normal vector in the case of the length parameterization of the curve.

$\boldsymbol{\nu}$ - normal vector in the case of both, arbitrary and length parameterizations of the curve.

$a_{i j}, h_{i j}$ - components of the metrics resp. of the curvature tensor in the case of an arbitrary parameterization of the curve.

For geometrical applications of the covariant derivation we refer to [1], and for mechanical applications to [11].

\subsection{Definition of penetration. Closest point procedure.}

Let the boundary of the master body be a smooth curve, parameterized by the parameter $\xi: \boldsymbol{\rho}=\boldsymbol{\rho}(\xi)$. The vector $\mathbf{r}_{s}$ describes the location of a slave point $\mathbf{S}$, see Fig. 2. Then the problem to find the shortest distance between the curve $\boldsymbol{\rho}(\xi)$ and the slave point $\mathbf{S}$ is defined via the minimum of the function:

$$
F:=\left\|\mathbf{r}_{s}-\boldsymbol{\rho}(\xi)\right\| \longrightarrow \min .
$$

The necessary condition for the minimum is the requirement of the first derivative to be zero: 


$$
F^{\prime}=\left(\mathbf{r}_{s}-\boldsymbol{\rho}(\xi)\right) \cdot \frac{d \boldsymbol{\rho}}{d \xi}=0 .
$$

Eqn. (2) is identical to the orthogonality condition between the vector $\mathbf{r}_{s}-$ $\boldsymbol{\rho}(\xi)$ and the tangent vector $\frac{d \boldsymbol{\rho}}{d \xi} \equiv \boldsymbol{\rho}_{\xi}$, and serves to define a projection point $\mathbf{C}$, see Fig. 2. The solution can be obtained e.g. by an iterative Newton scheme. For the latter the second derivative is necessary:

$$
F^{\prime \prime}=\left(\mathbf{r}_{s}-\boldsymbol{\rho}(\xi)\right) \cdot \frac{d^{2} \boldsymbol{\rho}}{d \xi^{2}}-\frac{d \boldsymbol{\rho}}{d \xi} \cdot \frac{d \boldsymbol{\rho}}{d \xi},
$$

which is finally shown to be positive to specify the minimum distance. The iterative scheme is then defined as:

$$
\left\{\begin{array}{l}
\Delta \xi=-F^{\prime} / F^{\prime \prime}=-\frac{\left(\mathbf{r}_{s}-\boldsymbol{\rho}\right) \cdot \boldsymbol{\rho}_{\xi}}{\left(\mathbf{r}_{s}-\boldsymbol{\rho}\right) \cdot \boldsymbol{\rho}_{\xi \xi}-\left(\boldsymbol{\rho}_{\xi} \cdot \boldsymbol{\rho}_{\xi}\right)} . \\
\xi^{(n+1)}=\xi^{(n)}+\Delta \xi
\end{array}\right.
$$

We will show in the finite implementation section, that for a $2 \mathrm{D}$ contact element with a linear approximation the general iterative scheme is reduced to an exact definition of the projection point.

\section{$2.32 \mathrm{D}$ contact kinematics}

In the $2 \mathrm{D}$ case contact bodies are bounded by plane curves, therefore, one can take advantage of their geometry. The geometrical properties of the contact quantities can be defined in a very straightforward manner, if we use the natural parameter length, i.e. $\boldsymbol{\rho}=\boldsymbol{\rho}(s)$ with $s=s(\xi)$. On the plane we define a curvilinear coordinate system associated with the curve by introducing two principal vectors as a basis: the tangent vector $\boldsymbol{\rho}_{\xi}=\frac{\partial \boldsymbol{\rho}}{\partial \xi}$ and the unit normal vector $\boldsymbol{\nu}$

$$
\mathbf{r}_{s}(\xi, \zeta)=\boldsymbol{\rho}(\xi)+\zeta \boldsymbol{\nu}(\xi)
$$

Looking at the following implementation of the algorithm in a FE program, the introduction of a natural parameter $s$ would lead to additional numerical effort, because the length of a boundary is changing during deformation. Thus, we will only show the geometrical properties using the parameter $s$, whereas for a finite element implementation we then turn to the Lagrangian coordinate $\xi$. As the local coordinate system is associated with the slave point $\mathbf{S}$, then 


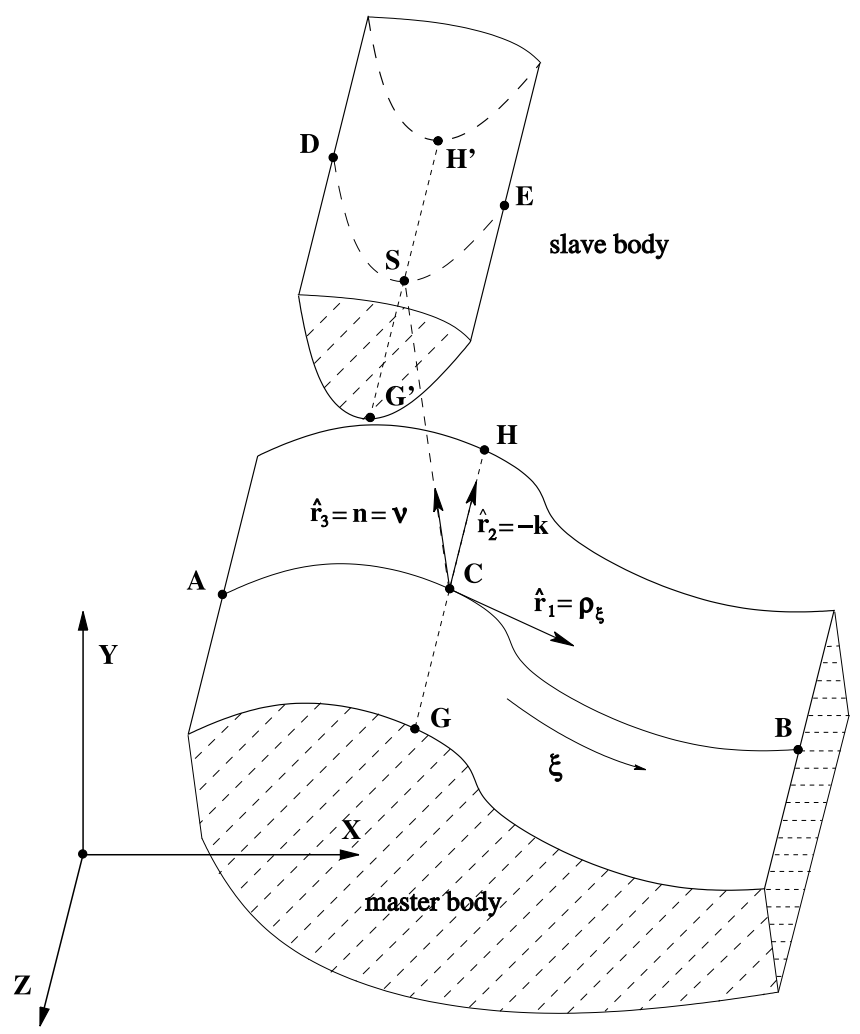

Figure 1: Two dimensional contact as a special case of three dimensional contact - contact between cylindrical surfaces with parallel axes $Z$. Local surface coordinate system on smooth master contact surface.

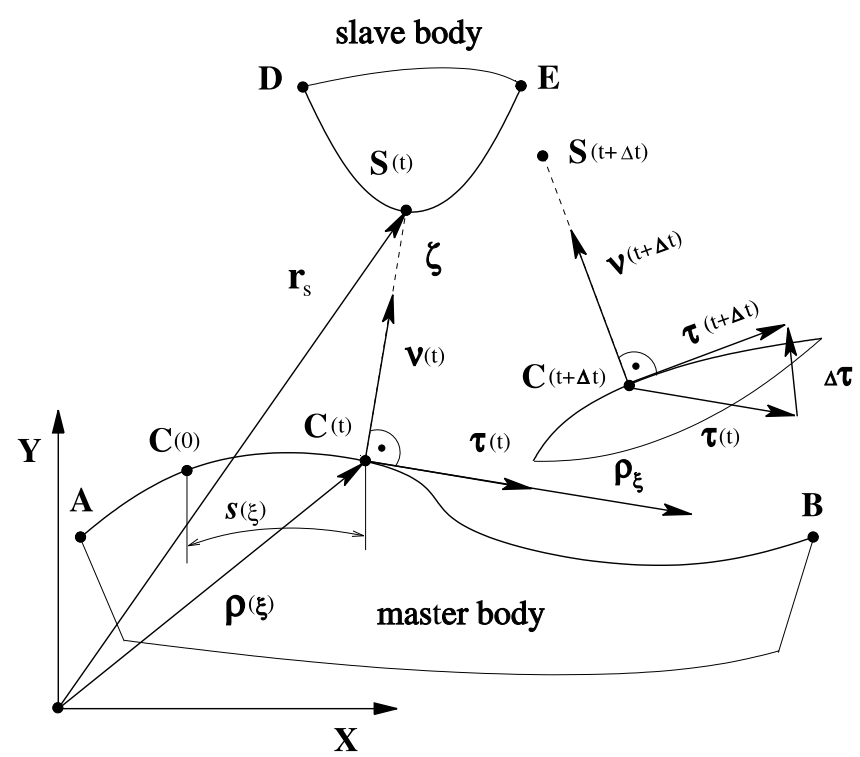

Figure 2: Two dimensional contact. Definitions. Contact boundaries are smooth curves in 2D.

the closest point procedure eqn. (2) is already fulfilled by this definition. The second coordinate $\zeta$ is an exact (not scaled!) value of penetration often also known as gap and used for the formulation of the non-penetrability condition.

The normal unit vector $\boldsymbol{\nu}$ in the case of arbitrary Lagrangian parameteriza- 
tions with $\xi$ can be defined via a cross product in a Cartesian coordinate system as:

$$
\boldsymbol{\rho}_{\xi}=\frac{\partial \boldsymbol{\rho}}{\partial \xi} ; \Longrightarrow \quad \boldsymbol{\nu}=\frac{\left[\mathbf{k} \times \boldsymbol{\rho}_{\xi}\right]}{\sqrt{\boldsymbol{\rho}_{\xi} \cdot \boldsymbol{\rho}_{\xi}}}
$$

where $\mathbf{k}$ is the third unit vector in this Cartesian coordinate system. The definition of the normal vector in eqn. (6) comes from the standard definition of the surface unit normal for cylindrical surfaces, see the contact between cylinders in Fig. 1, as

$$
\boldsymbol{\nu}=\frac{\left[\hat{\mathbf{r}}_{1} \times \hat{\mathbf{r}}_{2}\right]}{\left|\left[\hat{\mathbf{r}}_{1} \times \hat{\mathbf{r}}_{2}\right]\right|}, \quad \hat{\mathbf{r}}_{1}=\boldsymbol{\rho}_{\xi}, \quad \hat{\mathbf{r}}_{2}=\mathbf{k},
$$

where $\hat{\mathbf{r}}_{2}$ is a unit vector of the cylinder generatrix. The definition in eqn. (6) gives a set of covariant basis vectors $\boldsymbol{\rho}_{\xi}, \mathbf{k}$ for a cylindrical surface. The surface metric tensor can then be defined by the following matrix $a_{i j}$ :

$$
\left[a_{i j}\right]=\left[\begin{array}{cc}
\left(\boldsymbol{\rho}_{\xi} \cdot \boldsymbol{\rho}_{\xi}\right) & 0 \\
0 & 1
\end{array}\right]
$$

This matrix allows to define a contravariant basis for the cylindrical surface $\boldsymbol{\rho}^{1}, \boldsymbol{\rho}^{2}$, where only the first vector is changing its length:

$$
\left(\begin{array}{c}
\boldsymbol{\rho}^{1} \\
\boldsymbol{\rho}^{2}
\end{array}\right)=\left[\begin{array}{cc}
\left(\boldsymbol{\rho}_{\xi} \cdot \boldsymbol{\rho}_{\xi}\right) & 0 \\
0 & 1
\end{array}\right]^{-1}\left(\begin{array}{c}
\boldsymbol{\rho}_{\xi} \\
\boldsymbol{\nu}
\end{array}\right) \quad \Longrightarrow \quad \boldsymbol{\rho}^{1}=\frac{\boldsymbol{\rho}_{\xi}}{\left(\boldsymbol{\rho}_{\xi} \cdot \boldsymbol{\rho}_{\xi}\right)}, \quad \boldsymbol{\rho}^{2}=\boldsymbol{\nu}
$$

We note here, that the dot product of the covariant basis with the contravariant basis $\boldsymbol{\rho}^{1}, \boldsymbol{\rho}^{2}$ leads to a unit matrix (a mixed metrics tensor):

$$
\left[a_{i}^{j}\right]=\left[\left(\boldsymbol{\rho}_{i} \cdot \boldsymbol{\rho}^{j}\right)\right]=\left[\begin{array}{cc}
1 & 0 \\
0 & 1
\end{array}\right]
$$

\subsubsection{Derivatives of the basis vectors}

Derivatives of the covariant basis vectors $\boldsymbol{\rho}_{\xi}$ and $\boldsymbol{\nu}$ are necessary for the further formulation and for the linearization. The derivative of the tangent vector $\boldsymbol{\rho}_{\xi}$ can be expressed via the covariant basis vectors

$$
\boldsymbol{\rho}_{\xi \xi}:=\frac{\partial \boldsymbol{\rho}_{\xi}}{\partial \xi}=\Gamma \boldsymbol{\rho}_{\xi}+h_{11} \boldsymbol{\nu}
$$

where $\Gamma$ and $h_{11}$ are defined after taking a scalar product of eqn. (11) with $\boldsymbol{\rho}_{\xi}$ and $\boldsymbol{\nu}$ :

$$
\Gamma=\frac{\boldsymbol{\rho}_{\xi \xi} \cdot \boldsymbol{\rho}_{\xi}}{\boldsymbol{\rho}_{\xi} \cdot \boldsymbol{\rho}_{\xi}}, \quad h_{11}=\boldsymbol{\rho}_{\xi \xi} \cdot \boldsymbol{\nu}
$$


In order to compute the derivative of the unit normal $\boldsymbol{\nu}$, first, we have to take the derivative of the following identity:

$$
\frac{\partial}{\partial \xi}(\boldsymbol{\nu} \cdot \boldsymbol{\nu}=1) \quad \Longrightarrow \quad \frac{\partial \boldsymbol{\nu}}{\partial \xi} \cdot \boldsymbol{\nu}=0
$$

which leads to the orthogonality condition of the vectors $\boldsymbol{\nu}$ and $\frac{\partial \boldsymbol{\nu}}{\partial \xi}$. Thus, this derivative can be expressed via the tangent vector $\boldsymbol{\rho}_{\xi}$ as:

$$
\boldsymbol{\nu}_{\xi}=a \boldsymbol{\rho}_{\xi} .
$$

Then the scalar $a$ is obtained after taking the dot product of eqn. (14) and $\boldsymbol{\rho}_{\xi}$ :

$$
a=\left(\boldsymbol{\nu}_{\xi} \cdot \boldsymbol{\rho}_{\xi}\right) /\left(\boldsymbol{\rho}_{\xi} \cdot \boldsymbol{\rho}_{\xi}\right)=-\left(\boldsymbol{\rho}_{\xi \xi} \cdot \boldsymbol{\nu}\right) /\left(\boldsymbol{\rho}_{\xi} \cdot \boldsymbol{\rho}_{\xi}\right) \text {. }
$$

The last equation is obtained via the derivative of the equation $\boldsymbol{\rho}_{\xi} \cdot \boldsymbol{\nu}=0$. Finally, we get

$$
\boldsymbol{\nu}_{\xi}=-\frac{\left(\boldsymbol{\rho}_{\xi \xi} \cdot \boldsymbol{\nu}\right)}{\left(\boldsymbol{\rho}_{\xi} \cdot \boldsymbol{\rho}_{\xi}\right)} \boldsymbol{\rho}_{\xi}=-\frac{h_{11}}{a_{11}} \boldsymbol{\rho}_{\xi} .
$$

In eqns. (12) and (16) the scalar value $h_{11}=\left(\boldsymbol{\rho}_{\xi \xi} \cdot \boldsymbol{\nu}\right)$ is a curvature coefficient and $a_{11}=\left(\boldsymbol{\rho}_{\xi} \cdot \boldsymbol{\rho}_{\xi}\right)$ a metric coefficient for a cylindrical surface. Eqn. (16) represents the Weingarten formula and eqn. (11) resp. the Gauss-Codazzi formula for a cylindrical surface. Now, we can obtain the basis vectors for the coordinate system in eqn. (5):

$$
\begin{aligned}
& \mathbf{r}_{1}=\frac{\partial \mathbf{r}}{\partial \xi}=\left(1-\frac{h_{11}}{a_{11}} \zeta\right) \boldsymbol{\rho}_{\xi}, \\
& \mathbf{r}_{2}=\boldsymbol{\nu} .
\end{aligned}
$$

The geometrical properties in 2D contact are easily found, if they are reconsidered from the plane curve geometry with a natural parameterization. This parameterization is based on a length parameter $s$, i.e. $\boldsymbol{\rho}=\boldsymbol{\rho}(s)$. The length $s$ represents then the full path length passed by the projection point $C$ on the master curve during contact interaction, see Fig. 2. The tangent vector $\tau$ in this case has a unit length

$$
\boldsymbol{\tau}=\frac{d \boldsymbol{\rho}}{d s} .
$$

The relation between the parameter $\xi$ and the length parameter $s$ is defined as:

$$
d s=\sqrt{\left(\boldsymbol{\rho}_{\xi} \cdot \boldsymbol{\rho}_{\xi}\right)} d \xi .
$$

For the plane curve in natural parameterization the Serret-Frenet formulae are used to define derivatives of the basis vectors in analogy to eqns. (11) and (16):

$$
\frac{d \boldsymbol{\tau}}{d s}=\kappa \boldsymbol{\nu} ; \quad \frac{d \boldsymbol{\nu}}{d s}=-\kappa \boldsymbol{\tau},
$$


where $\kappa$ is a curvature of the curve. In this case the normal unit vector $\boldsymbol{\nu}$ is defined from eqn. (20) and is pointing into the convex part of a body. Then the curvature $\kappa$ can be computed from eqn. (20) by premultiplying the first equation with $\boldsymbol{\nu}$ and taking the chain rule into account:

$$
\kappa=\frac{d \boldsymbol{\tau}}{d s} \cdot \boldsymbol{\nu}=\frac{\left(\boldsymbol{\rho}_{\xi \xi} \cdot \boldsymbol{\nu}\right)}{\left(\boldsymbol{\rho}_{\xi} \cdot \boldsymbol{\rho}_{\xi}\right)}=\frac{h_{11}}{a_{11}}
$$

2.3.2 Covariant derivative of a tangent vector $\mathrm{T}$.

We define a tangent vector field $\mathbf{T}$ - later taken as a friction force - as a covariant vector field in the spatial coordinate system:

$$
\mathbf{T}(\xi)=\left.T(\xi) \mathbf{r}^{1}\right|_{\zeta=0}=T(\xi) \boldsymbol{\rho}^{1}=T(\xi) \frac{\boldsymbol{\rho}_{\xi}}{\left(\boldsymbol{\rho}_{\xi} \cdot \boldsymbol{\rho}_{\xi}\right)} .
$$

The definition in the form of eqn. (22) has the advantage that the weak form - used later - becomes rather simple. The absolute value of the covariant vector eqn. (22) is computed as:

$$
\|\mathbf{T}(\xi)\|=\frac{|T(\xi)|}{\left\|\boldsymbol{\rho}_{\xi}\right\|}=\frac{|T(\xi)|}{\sqrt{a_{11}}} .
$$

The full time derivative of this vector field is determined taking the changing metric into account and then considering its value at $\zeta=0$ :

$$
\left.\left.\frac{d \mathbf{T}}{d t}\right|_{\zeta=0}=[\underbrace{\left(\frac{\partial T}{\partial t}+\frac{\partial T}{\partial \xi} \dot{\xi}\right)}_{\frac{d T}{d t}} \mathbf{r}^{1}+T\left(\dot{\xi} \frac{\partial \mathbf{r}^{1}}{\partial \xi}+\dot{\zeta} \frac{\partial \mathbf{r}^{1}}{\partial \zeta}\right))\right]_{\zeta=0} .
$$

The derivative is expressed then via the contravariant basis vectors as $\boldsymbol{\rho}^{1}, \boldsymbol{\rho}^{2}$

$$
\left.\frac{d \mathbf{T}}{d t}\right|_{\zeta=0}=\frac{D_{1} T}{d t} \boldsymbol{\rho}^{1}+\frac{D_{2} T}{d t} \boldsymbol{\rho}^{2} .
$$

Here a full time derivative in the covariant form is introduced. Its value on the tangent line is computed using the scalar product with $\boldsymbol{\rho}_{\xi}$ as (see Appendix):

$$
\frac{D_{1} T}{d t}:=\left(\frac{d \mathbf{T}}{d t} \cdot \boldsymbol{\rho}_{\xi}\right)_{\zeta=0}=\frac{d T}{d t}-\frac{\boldsymbol{\rho}_{\xi \xi} \cdot \boldsymbol{\rho}_{\xi}}{\left(\boldsymbol{\rho}_{\xi} \cdot \boldsymbol{\rho}_{\xi}\right)} \dot{\xi}+\frac{h_{11}}{a_{11}} \dot{\zeta} .
$$

The second term on the right hand side contains the Christoffel symbol and the last term contains the curvature for a cylindrical surface. 


\subsubsection{Convective velocities}

An important part for the formulation as well as for the linearization of the weak form is a time derivative of the vector of a slave point $\mathbf{S}$ in eqn. (5)

$$
\frac{d \mathbf{r}_{\mathrm{s}}}{d t}=\frac{\partial \boldsymbol{\rho}}{\partial t}+\dot{\xi} \boldsymbol{\rho}_{\xi}+\zeta\left(\frac{\partial \boldsymbol{\nu}}{\partial t}+\dot{\xi} \frac{\partial \boldsymbol{\nu}}{\partial \xi}\right)+\dot{\zeta} \boldsymbol{\nu}
$$

With $\mathbf{v}_{s}=\frac{d \mathbf{r}_{s}}{d t}$ as the absolute velocity of the slave point $\mathbf{S}$ resp. $\mathbf{v}=\frac{\partial \boldsymbol{\rho}}{\partial t}$ as the velocity of its projection on the master surface. The dot product with the normal vector $\boldsymbol{\nu}$ leads to the rate of the penetration

$$
\dot{\zeta}=\left(\mathbf{v}_{s}-\mathbf{v}\right) \cdot \boldsymbol{\nu}
$$

Considering a value of the convective tangent velocity $\dot{\xi}$ on the tangent line, i.e. at $\zeta=0$, we need the dot product of eqn. (26) with $\boldsymbol{\rho}_{\xi}$ :

$$
\dot{\xi}=\frac{\left(\mathbf{v}_{s}-\mathbf{v}\right) \cdot \boldsymbol{\rho}_{\xi}}{\left(\boldsymbol{\rho}_{\xi} \cdot \boldsymbol{\rho}_{\xi}\right)} .
$$

In the case of a length parameterization with $s=\xi$, eqn. (28) leads to the following convective velocity $\dot{s}$ :

$$
\dot{s}=\left(\mathbf{v}_{s}-\mathbf{v}\right) \cdot \boldsymbol{\tau} .
$$

From the kinematical equation (26) we can obtain an equation for the variations by changing the time derivative operator into the variation operator $\delta$. This equation is also considered on the tangent line, i.e. at $\zeta=0$ :

$$
\delta \mathbf{r}_{s}-\delta \boldsymbol{\rho}=\delta \xi \boldsymbol{\rho}_{\xi}+\delta \zeta \boldsymbol{\nu}
$$

Eqn. (30) gives a variation of the displacement field for the expression of the virtual work of contact tractions on the contact surface.

\subsubsection{Evolution equations for contact tractions}

The evolution equations can be regarded as rate equations for the contact tractions. The contact traction vector $\mathbf{R}_{s}$ is defined for the slave point $s$ in the local coordinate system on the master curve in the covariant form as:

$$
\mathbf{R}_{s}=T \boldsymbol{\rho}^{1}+N \boldsymbol{\rho}^{2}=\mathbf{T}+N \boldsymbol{\rho}^{2}=T \frac{\boldsymbol{\rho}_{\xi}}{\left(\boldsymbol{\rho}_{\xi} \cdot \boldsymbol{\rho}_{\xi}\right)}+N \boldsymbol{\nu} .
$$

For the normal traction $N$, the following regularized equation is applied

$$
N=\epsilon_{N}\langle\zeta\rangle
$$


where $\epsilon_{N}$ is a penalty parameter for the normal interaction and \langle\rangle are Macauley brackets in the form

$$
\langle\zeta\rangle=\left\{\begin{array}{ll}
0, & \text { if } \zeta>0 \\
\zeta, & \text { if } \zeta \leq 0
\end{array} .\right.
$$

According to eqn. (27), the rate of a normal traction can be computed as

$$
\dot{N}=\epsilon_{N} \dot{\zeta} H(-\zeta)=\epsilon_{N} H(-\zeta)\left(\mathbf{v}_{s}-\mathbf{v}\right) \cdot \boldsymbol{\nu},
$$

where $H(-\zeta)$ is the Heaviside function.

As a reasonable equation for the regularization of the tangent traction vector $\mathbf{T}$ we choose a proportional relation between the full time derivative $\frac{d \mathbf{T}}{d t}$ and the relative velocity vector expressed on the tangent line $\zeta=0$ :

$$
\frac{D_{1} T}{d t} \boldsymbol{\rho}^{1}=-\epsilon_{T} \dot{\xi} \boldsymbol{\rho}_{\xi}
$$

in component form written as

$$
\frac{D_{1} T}{d t}=-\epsilon_{T} \dot{\xi}\left(\boldsymbol{\rho}_{\xi} \cdot \boldsymbol{\rho}_{\xi}\right)
$$

where $\epsilon_{T}$ is a penalty parameter for the tangential interaction. Applying the results from eqn. (25) leads to the evolution equations in the form of covariant derivatives:

$$
\frac{d T}{d t}=-\epsilon_{T}\left(\boldsymbol{\rho}_{\xi} \cdot \boldsymbol{\rho}_{\xi}\right) \dot{\xi}+\frac{\boldsymbol{\rho}_{\xi \xi} \cdot \boldsymbol{\rho}_{\xi}}{\left(\boldsymbol{\rho}_{\xi} \cdot \boldsymbol{\rho}_{\xi}\right)} \dot{\xi}-\frac{h_{11}}{a_{11}} \dot{\zeta} .
$$

which is used to compute a trial tangent traction.

\section{Weak formulation in the spatial coordinate system.}

Next we consider the contact tractions $\mathbf{R}_{s}$ and $\mathbf{R}_{m}$ on both the slave and the master contact curves with corresponding lengths $l_{s}$ and $l_{m}$ in the current configuration. Let $\delta \mathbf{u}_{s}$ resp. $\delta \mathbf{u}_{m}$ be variations of the displacement field on the curves $l_{s}$ resp. $l_{m}$, then the work of the contact forces is determined in the following integral

$$
\delta W_{c}=\int_{l_{s}} \mathbf{R}_{s} \cdot \delta \mathbf{u}_{s} d l_{s}+\int_{l_{m}} \mathbf{R}_{m} \cdot \delta \mathbf{u}_{m} d l_{m},
$$

which must be added to the global work of the internal and external forces. Due to equilibrium at the contact boundary $\mathbf{R}_{s} d l_{s}=-\mathbf{R}_{m} d l_{m}$, equation (37) can be also written as 


$$
\delta W_{c}=\int_{l_{s}} \mathbf{R}_{s} \cdot\left(\delta \mathbf{u}_{s}-\delta \mathbf{u}_{m}\right) d l_{s} .
$$

The integral in (38) is considered in the local coordinate system. We redefine now the variations $\delta \mathbf{u}_{s}=\delta \mathbf{r}_{s}$ for a slave point and $\delta \mathbf{u}_{m}=\delta \boldsymbol{\rho}$ for a projection of the slave point onto the master curve.

Substituting the variation $\delta \mathbf{u}_{s}-\delta \mathbf{u}_{m}=\delta \mathbf{r}_{s}-\delta \boldsymbol{\rho}$ from eqn. (30) and also the full contact traction vector eqn. (31) into the integral (38) we obtain

$$
\delta W_{c}=\int_{l}(N \delta \zeta+T \delta \xi) d l .
$$

A closer look reveals that the contact integral (39) contains the work of the contact tractions $T$ and $N$ defined on the master contact curve and is computed along the slave curve $l \equiv l_{s}$.

\section{Linearization process.}

Here, we show the derivation of the normal contact matrices using the geometry of plane curves. For all other results, we give a sketch of the linearization procedure with a comparative discussion of results available in the literature, in order to avoid the repetition of complicated mathematics.

\subsection{Necessary operations. Linearization of convective variations.}

Since in the contact integral the linearization of the contact tractions is directly given by the evolution equations, it is only necessary to find derivatives of the convective variations $\delta \zeta$ and $\delta \xi$ to fulfill all steps in the preparation for further forms.

\subsubsection{Linearization of $\delta \zeta$.}

The result will be obtained assuming a natural parameterization of the corresponding boundary curve.

$$
\begin{gathered}
\frac{d}{d t} \delta \zeta=\frac{d}{d t}\left[\left(\delta \mathbf{r}_{s}-\delta \boldsymbol{\rho}\right) \cdot \boldsymbol{\nu}\right]= \\
=\frac{\partial\left(\delta \mathbf{r}_{s}-\delta \boldsymbol{\rho}\right)}{\partial s} \cdot \boldsymbol{\nu} \dot{s}+\left(\delta \mathbf{r}_{s}-\delta \boldsymbol{\rho}\right) \cdot \frac{\partial \boldsymbol{\nu}}{\partial t}+\left(\delta \mathbf{r}_{s}-\delta \boldsymbol{\rho}\right) \cdot \frac{\partial \boldsymbol{\nu}}{\partial s} \dot{s}
\end{gathered}
$$


The first term can be rewritten, taking into account eqn. (29) for a convective velocity in the case of a natural parameterization, as follows:

$$
\delta \underbrace{\frac{\partial\left(\mathbf{r}_{s}-\boldsymbol{\rho}\right)}{\partial s}}_{-\boldsymbol{\tau}} \cdot \boldsymbol{\nu} \dot{s}=-(\delta \boldsymbol{\tau} \cdot \boldsymbol{\nu}) \underbrace{\left(\left(\mathbf{v}_{s}-\mathbf{v}\right) \cdot \boldsymbol{\tau}\right)}_{\dot{s}}=-\delta \boldsymbol{\tau} \cdot(\boldsymbol{\nu} \otimes \boldsymbol{\tau})\left(\mathbf{v}_{s}-\mathbf{v}\right) .
$$

In order to rewrite the second term, we have to take first a partial time derivative of the orthogonality condition:

$$
\boldsymbol{\tau} \cdot \boldsymbol{\nu}=0 \quad \Longrightarrow \quad \frac{\partial(\boldsymbol{\tau} \cdot \boldsymbol{\nu})}{\partial t}=\frac{\partial^{2} \boldsymbol{\rho}}{\partial s \partial t} \cdot \boldsymbol{\nu}+\frac{\partial \boldsymbol{\nu}}{\partial t} \cdot \boldsymbol{\tau}=0,
$$

leading to the expression

$$
\frac{\partial \boldsymbol{\nu}}{\partial t} \cdot \boldsymbol{\tau}=-\frac{\partial \mathbf{v}}{\partial s} \cdot \boldsymbol{\nu}
$$

From the other side, using the unity condition of the vector $\boldsymbol{\nu}$, we can express the time derivative in terms of the tangent vector $\boldsymbol{\tau}$ by analogy to eqns. (14), (15), as

and substituting eqn. (43) we obtain

$$
\frac{\partial \boldsymbol{\nu}}{\partial t}=a \boldsymbol{\tau}=\left(\boldsymbol{\tau} \cdot \frac{\partial \boldsymbol{\nu}}{\partial t}\right) \boldsymbol{\tau}
$$

$$
\frac{\partial \boldsymbol{\nu}}{\partial t}=-\left(\frac{\partial \mathbf{v}}{\partial s} \cdot \boldsymbol{\nu}\right) \boldsymbol{\tau}
$$

Eqn. (45) allows to transform the second term in eqn. (40) as follows

$$
\left(\delta \mathbf{r}_{s}-\delta \boldsymbol{\rho}\right) \cdot \frac{\partial \boldsymbol{\nu}}{\partial t}=-\left(\delta \mathbf{r}_{s}-\delta \boldsymbol{\rho}\right) \cdot\left(\frac{\partial \mathbf{v}}{\partial s} \cdot \boldsymbol{\nu}\right) \boldsymbol{\tau}=
$$

introducing a tensor product $\boldsymbol{\tau} \otimes \boldsymbol{\nu}$ in order to transform a dot product

$$
=-\left(\delta \mathbf{r}_{s}-\delta \boldsymbol{\rho}\right) \cdot(\boldsymbol{\tau} \otimes \boldsymbol{\nu}) \frac{\partial \mathbf{v}}{\partial s}=-\left(\delta \mathbf{r}_{s}-\delta \boldsymbol{\rho}\right) \cdot(\boldsymbol{\tau} \otimes \boldsymbol{\nu}) \frac{\partial \boldsymbol{\tau}}{\partial t} .
$$

The last term in eqn. (47) is obtained reversing the order of differentiation as

$$
\frac{\partial \mathbf{v}}{\partial s}=\frac{\partial}{\partial s} \frac{\partial \boldsymbol{\rho}}{\partial t}=\frac{\partial}{\partial t} \frac{\partial \boldsymbol{\rho}}{\partial s}=\frac{\partial \boldsymbol{\tau}}{\partial t}
$$

The third term in (40) is reorganized into a tensor form with a second SerretFrenet formula and with equation (29) for the convective velocity $\dot{s}$ :

$$
\left(\delta \mathbf{r}_{s}-\delta \boldsymbol{\rho}\right) \cdot \frac{\partial \boldsymbol{\nu}}{\partial s} \dot{s}=-\left(\delta \mathbf{r}_{s}-\delta \boldsymbol{\rho}\right) \cdot \kappa \boldsymbol{\tau} \otimes \boldsymbol{\tau}\left(\mathbf{v}_{s}-\mathbf{v}\right)
$$

Therefore, combining eqn. (41), (46) and (49), we obtain a final formula for the linearization of $\delta \zeta$ :

$$
\frac{d}{d t} \delta \zeta=-\left(\delta \boldsymbol{\tau} \cdot \boldsymbol{\nu} \otimes \boldsymbol{\tau}\left(\mathbf{v}_{s}-\mathbf{v}\right)+\left(\delta \mathbf{r}_{s}-\delta \boldsymbol{\rho}\right) \cdot \boldsymbol{\tau} \otimes \boldsymbol{\nu} \frac{\partial \boldsymbol{\tau}}{\partial t}\right)-\left(\delta \mathbf{r}_{s}-\delta \boldsymbol{\rho}\right) \cdot \kappa \boldsymbol{\tau} \otimes \boldsymbol{\tau}\left(\mathbf{v}_{s}-\mathbf{v}\right)
$$


4.1.2 Linearization of the convective variation $\delta \xi$.

The linearization of the convective variations $\delta \xi^{i}$ in a 3D formulation is the most complicated part of the process. First, see Parisch [14], Laursen and Simo [9], Wriggers [24] the convective variations $\delta \xi^{i}$ were introduced via an iterative Newton scheme, see eqn. (4). A kinematical definition of $\delta \xi^{i}$ can be found in the books of Wriggers [26] and Laursen [10]. The full linearization of these terms combined with the contact integral defined only on the surface led to an artificial non-symmetry of the tangent matrix for the sticking case, which was mentioned in Laursen and Simo [9]. Wriggers [26] could avoid this by looking at it as a mesh tying procedure. In [7] the variations of $\delta \xi^{i}$ were defined kinematically and expressed on the tangent plane of the contact surface. In addition, the linearization process was performed in the covariant form on the tangent plane. For the sticking case this leads directly to a symmetric matrix and allows to avoid the artificial non-symmetry.

Here only the main points of the linearization process are depicted, for the full derivation we refer to [7].

1. The convective variations are defined on the tangent plane of the spatial coordinate system via consideration of the slave point velocity as $\dot{\xi}^{j}=$ $a^{i j}\left(\mathbf{v}_{s}-\mathbf{v}\right) \cdot \boldsymbol{\rho}_{i}$.

2. During the linearization of $\delta \xi^{i}$ the derivative of the metric tensor is obtained as derivative of the spatial metric tensor considering its value on the tangent plane.

The final result for the 3D case is then:

$$
\begin{gathered}
\frac{d}{d t}\left(\delta \xi^{i}\right)= \\
=-\left(\delta \mathbf{r}_{s}-\delta \boldsymbol{\rho}\right) a^{i l} a^{j k} \boldsymbol{\rho}_{k} \otimes \boldsymbol{\rho}_{l} \mathbf{v}_{j}-\delta \boldsymbol{\rho}_{, j} a^{i k} a^{j l} \boldsymbol{\rho}_{k} \otimes \boldsymbol{\rho}_{l}\left(\mathbf{v}_{s}-\mathbf{v}\right) \\
+h^{i j}\left(\delta \mathbf{r}_{s}-\delta \boldsymbol{\rho}\right) \cdot\left(\boldsymbol{\rho}_{j} \otimes \mathbf{n}+\mathbf{n} \otimes \boldsymbol{\rho}_{j}\right)\left(\mathbf{v}_{s}-\mathbf{v}\right)+ \\
+h_{n}^{i} \dot{\xi}^{3} \delta \xi^{n}-\Gamma_{k j}^{i} \dot{\xi}^{j} \delta \xi^{k} .
\end{gathered}
$$

The reduction into the specific plane geometry in the current contribution leads to:

$$
\begin{gathered}
\frac{d}{d t}(\delta \xi)= \\
=-\frac{\left(\delta \mathbf{r}_{s}-\delta \boldsymbol{\rho}\right) \cdot \boldsymbol{\rho}_{\xi} \otimes \boldsymbol{\rho}_{\xi} \mathbf{v}_{j}+\delta \boldsymbol{\rho}_{\xi} \cdot \boldsymbol{\rho}_{\xi} \otimes \boldsymbol{\rho}_{\xi}\left(\mathbf{v}_{s}-\mathbf{v}\right)}{\left(\boldsymbol{\rho}_{\xi} \cdot \boldsymbol{\rho}_{\xi}\right)^{2}}
\end{gathered}
$$




$$
\begin{gathered}
+\frac{\left(\boldsymbol{\rho}_{\xi \xi} \cdot \boldsymbol{\nu}\right)}{\left(\boldsymbol{\rho}_{\xi} \cdot \boldsymbol{\rho}_{\xi}\right)^{2}}\left(\delta \mathbf{r}_{s}-\delta \boldsymbol{\rho}\right) \cdot\left(\boldsymbol{\rho}_{\xi} \otimes \boldsymbol{\nu}+\boldsymbol{\nu} \otimes \boldsymbol{\rho}_{\xi}\right)\left(\mathbf{v}_{s}-\mathbf{v}\right)+ \\
+\frac{h_{11}}{a_{11}} \dot{\zeta} \delta \xi-\frac{\boldsymbol{\rho}_{\xi \xi} \cdot \boldsymbol{\rho}_{\xi}}{\left(\boldsymbol{\rho}_{\xi} \cdot \boldsymbol{\rho}_{\xi}\right)} \dot{\xi} \delta \xi
\end{gathered}
$$

The non-symmetric part in eqn. (52c) is intentionally kept in untransformed form, because it will give a zero in sum with similar terms in the evolution equation (36) during the forthcoming linearization.

\subsection{Tangent matrices}

We derive the tangent matrix for the normal part in the case of a natural parameterization. In order to avoid the complexity for the sticking - sliding cases for the tangential part, the derivation is given as a reduction of the $3 \mathrm{D}$ case.

\subsubsection{Tangent matrix for the normal part}

The normal part is defined by the following integral:

$$
\delta W_{c}^{N}=\int_{l} N \delta \zeta d l
$$

The integral is computed over the slave surface $l$, while all functions are defined on the master surface. Thus, a linearization of $d l$ is not necessary within the process:

$$
\begin{gathered}
D\left(\delta W_{c}^{N}\right)= \\
\int_{l}\left(\frac{d N}{d t} \delta \zeta+N \frac{d \delta \zeta}{d t}\right) d l=
\end{gathered}
$$

then the application of eqn. (33) and eqn. (50) leads to

$$
\begin{gathered}
=\int_{l} \epsilon_{N}\left(\delta \mathbf{r}_{s}-\delta \boldsymbol{\rho}\right) \cdot(\boldsymbol{\nu} \otimes \boldsymbol{\nu})\left(\mathbf{v}_{s}-\mathbf{v}\right) d l- \\
-\int_{l} \epsilon_{N} \zeta\left(\delta \boldsymbol{\tau} \cdot(\boldsymbol{\nu} \otimes \boldsymbol{\tau})\left(\mathbf{v}_{s}-\mathbf{v}\right)+\left(\delta \mathbf{r}_{s}-\delta \boldsymbol{\rho}\right) \cdot(\boldsymbol{\tau} \otimes \boldsymbol{\nu}) \frac{\partial \boldsymbol{\tau}}{\partial t}\right) d l- \\
-\int_{l} \epsilon_{N} \zeta \kappa\left(\delta \mathbf{r}_{s}-\delta \boldsymbol{\rho}\right) \cdot(\boldsymbol{\tau} \otimes \boldsymbol{\tau})\left(\mathbf{v}_{s}-\mathbf{v}\right) d l
\end{gathered}
$$

\section{Remark.}

The contact matrix obtained via eqn. (54) is computed only for the case $\zeta<0$ - this simplification allows us to exclude the usage of the Heaviside function. 
The form in natural coordinates allows a simple geometrical interpretation of each part in eqn. (54) and even allows to determine situations where some of them are zero. The first part eqn. (54a) is called main part and defines the constitutive relation for normal contact conditions. The second part eqn. (54b) is called rotational part and defines the geometrical stiffness due to the rotation of the tangent vector of the master curve. It disappears when a master segment is moving in parallel, because only in this case the derivative of a unit vector $\boldsymbol{\tau}$ becomes zero, see Fig. 2. The third part eqn. (54c) is called curvature part. This part disappears when the curvature $\kappa$ of a master segment is zero, i.e. in the case of linear approximations of the master segment.

\subsubsection{Tangent matrix for tangential traction}

The part of the contact integral which includes the effect of the tangential interaction is given as:

$$
\delta W_{c}^{T}=\int_{l} T \delta \xi d l
$$

The linearized equation has to be subdivided into a part for sticking and another part for sliding, which differ concerning the return-mapping scheme.

Sticking. In this case, the tangential force $T$ has to be computed from the solution of the evolution equation eqn. (36), e.g. via the backward Euler scheme. The simplest case with linear approximations will be presented in the following section concerning the finite element implementation.

Sticking is fulfilled according to Coulomb's friction law, i.e. the inequality $\|\mathbf{T}\| \leq \mu|N|$ has to be valid in each load step. The linearized contact integral has then the following form:

$$
\begin{gathered}
D_{v}\left(\delta W_{c}^{T}\right)=\int_{l}\left(\frac{d T}{d t} \delta \xi+T \frac{d \delta \xi}{d t}\right) d l= \\
-\int_{l} \frac{\varepsilon_{T}}{\left(\boldsymbol{\rho}_{\xi} \cdot \boldsymbol{\rho}_{\xi}\right)}\left(\delta \mathbf{r}_{s}-\delta \boldsymbol{\rho}\right) \cdot \boldsymbol{\rho}_{\xi} \otimes \boldsymbol{\rho}_{\xi}\left(\mathbf{v}_{s}-\mathbf{v}\right) d l \\
-\int_{l} \frac{T}{\left(\boldsymbol{\rho}_{\xi} \cdot \boldsymbol{\rho}_{\xi}\right)^{2}}\left[\left(\delta \mathbf{r}_{s}-\delta \boldsymbol{\rho}\right) \cdot \boldsymbol{\rho}_{\xi} \otimes \boldsymbol{\rho}_{\xi} \mathbf{v}_{\xi}+\delta \boldsymbol{\rho}_{\xi} \cdot \boldsymbol{\rho}_{\xi} \otimes \boldsymbol{\rho}_{\xi}\left(\mathbf{v}_{s}-\mathbf{v}\right)\right] d l \\
+\int_{l} \frac{T h_{11}}{\left(\boldsymbol{\rho}_{\xi} \cdot \boldsymbol{\rho}_{\xi}\right)^{2}}\left(\delta \mathbf{r}_{s}-\delta \boldsymbol{\rho}\right) \cdot\left(\boldsymbol{\rho}_{\xi} \otimes \boldsymbol{\nu}+\boldsymbol{\nu} \otimes \boldsymbol{\rho}_{\xi}\right)\left(\mathbf{v}_{s}-\mathbf{v}\right) d l
\end{gathered}
$$


Sliding. If sliding is detected, i.e. if $\|\mathbf{T}\|>\mu|N|$, then the sliding force is computed according to Coulomb's friction law. We also keep a covariant form:

with

$$
T^{s l}=\mu|N| \frac{T_{t r}}{\left\|\mathbf{T}_{t r}\right\|}=\mu|N|\left\|\boldsymbol{\rho}_{\xi}\right\| \operatorname{sgn}\left(T_{t r}\right),
$$

$$
\left\|\boldsymbol{\rho}_{\xi}\right\|=\left(\boldsymbol{\rho}_{\xi} \cdot \boldsymbol{\rho}_{\xi}\right)^{1 / 2}=\sqrt{a_{11}} \text {. }
$$

The linearized contact integral gets the following form:

$$
\begin{gathered}
D_{v}\left(\delta W_{c}^{T}\right)= \\
-\int_{l} \frac{\epsilon_{N} \mu \operatorname{sgn}\left(T_{t r}\right)}{\left(\boldsymbol{\rho}_{\xi} \cdot \boldsymbol{\rho}_{\xi}\right)^{1 / 2}}\left(\delta \mathbf{r}_{s}-\delta \boldsymbol{\rho}\right) \cdot\left(\boldsymbol{\rho}_{\xi} \otimes \boldsymbol{\nu}\right)\left(\mathbf{v}_{s}-\mathbf{v}\right) d l \\
-\int_{l} \frac{\mu|N| \operatorname{sgn}\left(T_{t r}\right)}{\left(\left(\boldsymbol{\rho}_{\xi} \cdot \boldsymbol{\rho}_{\xi}\right)^{3 / 2}\right.}\left(\left(\mathbf{r}_{s}-\delta \boldsymbol{\rho}\right) \cdot \boldsymbol{\rho}_{\xi} \otimes \boldsymbol{\rho}_{\xi} \mathbf{v}_{\xi}+\delta \boldsymbol{\rho}_{\xi} \cdot \boldsymbol{\rho}_{\xi} \otimes \boldsymbol{\rho}_{\xi}\left(\mathbf{v}_{s}-\mathbf{v}\right)\right) d l \\
+\int_{l} \frac{\mu h_{11}|N| \operatorname{sgn}\left(T_{t r}\right)}{\left(\boldsymbol{\rho}_{\xi} \cdot \boldsymbol{\rho}_{\xi}\right)^{3 / 2}}\left(\delta \mathbf{r}_{s}-\delta \boldsymbol{\rho}\right) \cdot\left(2 \boldsymbol{\rho}_{\xi} \otimes \boldsymbol{\nu}+\boldsymbol{\nu} \otimes \boldsymbol{\rho}_{\xi}\right)\left(\mathbf{v}_{s}-\mathbf{v}\right) d l
\end{gathered}
$$

The non-symmetric part eqn. (58c) now is resulting from the last term of the evolution equation (36).

\section{Remark:}

The derivations for the two dimensional case allow to describe all parts of the tangent matrix and to find all cases, when some of them become zero. The main parts, eqns. (56a) and (58a), the so-called constitutive parts, contain a penalty parameter and describe the stiffness of the contact interaction due to the chosen interface model. This is based on an allowable elastic deformation due to the regularization in the case of sticking resp. due to the applied sliding force $\mu|N|$ in the tangential direction in the case of sliding. The rotational parts eqns. (56b) and (58b), contain a metric coefficient $a_{11}=\left(\boldsymbol{\rho}_{\xi} \cdot \boldsymbol{\rho}_{\xi}\right)$ and a vector $\boldsymbol{\rho}_{\xi}$. A metric coefficient is a measure of the tensile deformation of the contact master line, e.g. a component of the Cauchy-Green tensor for the contact line can be written as $\varepsilon_{11}=\left(a_{11}-1\right) / 2$. The vector $\boldsymbol{\rho}_{\xi}$ is a measure of the rotation of the master segment, which becomes obvious if the length is chosen as a coordinate $s=\xi$. In this case we find $\delta \boldsymbol{\rho}_{\xi}=\delta \boldsymbol{\tau}$ and $\mathbf{v}_{\xi}=\frac{\partial \boldsymbol{\tau}}{\partial t}$. The vector $\boldsymbol{\tau}$ is a unit vector, therefore the vectors $\delta \boldsymbol{\tau}$ and $\frac{\partial \boldsymbol{\tau}}{\partial t}$ are describing the rotation of the unit vector $\boldsymbol{\tau}$ (see Fig. 2). It is identical to zero only in the case of parallel motions. Thus, the rotational part is obviously negligible in the case of small deformations and small rotations of the master line. The curvature parts eqns. (56c) and (58c) describe the stiffness of the contact interaction due to the curvature of the contact surface. If the surface has zero curvature or is approximated by linear elements, then the curvature part becomes zero. 


\section{$5 \quad$ Finite element implementation}

The structure of all parts of the tangent matrix is algorithmic. It is sufficient for the discretization to define only approximations of a relative displacement vector $\left(\mathbf{r}_{s}-\boldsymbol{\rho}\right)$ and its derivative with respect to $\xi$. Following the standard iso-parametric technique as for finite elements, we consider a contact surface element with the same order of approximation for the geometry as for the displacement field. The boundary curve can be given with any curve description (spline, NURB, etc.). For simplicity, we consider only the node-to-segment contact approach. Let a boundary curve or a master segment of it be defined by $n$ nodes with $\mathbf{x}^{(1)}, \mathbf{x}^{(2)}, \ldots, \mathbf{x}^{(n)}$; and $\mathbf{x}^{(n+1)}$ for a slave node. A standard grouping of a displacement vector can then be written as

$$
\mathbf{u}^{T}=\left\{u_{1}^{(1)}, u_{2}^{(1)}, u_{1}^{(2)}, u_{2}^{(2)}, \ldots, u_{1}^{(n)}, u_{2}^{(n)}, u_{1}^{(n+1)}, u_{2}^{(n+1)}\right\}^{T},
$$

where the first $n$ nodes resp. $2 n$ displacements belong to the master surface, while the $(n+1)$ 'th term is belonging to the "slave" node resp. is describing the "slave" displacements.

We introduce a matrix of shape functions $\mathbf{A}$

$$
\mathbf{A}=\left[\begin{array}{ccccccccccc}
-N_{1} & 0 & 0 & -N_{2} & 0 & 0 & \ldots & -N_{n} & 0 & 1 & 0 \\
0 & -N_{1} & 0 & 0 & -N_{2} & 0 & \ldots & 0 & -N_{n} & 0 & 1
\end{array}\right]
$$

where $N_{i}, i=1,2, \ldots, n$ are shape functions. The matrix of the derivatives of the shape functions $\mathbf{A}^{\prime}$ is defined then as

$$
\mathbf{A}^{\prime}=-\left[\begin{array}{ccccccccccc}
N_{1}^{\prime} & 0 & 0 & N_{2}^{\prime} & 0 & 0 & \ldots & N_{n}^{\prime} & 0 & 0 & 0 \\
0 & N_{1}^{\prime} & 0 & 0 & N_{2}^{\prime} & 0 & \ldots & 0 & N_{n}^{\prime} & 0 & 0
\end{array}\right]
$$

The relative vector of variations $\left(\delta \mathbf{r}_{s}-\delta \boldsymbol{\rho}\right)$ and the relative velocity vector $\left(\mathbf{v}_{s}-\mathbf{v}\right)$ are then written as

$$
\delta \mathbf{r}_{s}-\delta \boldsymbol{\rho}=\mathbf{A} \delta \mathbf{u}, \quad \mathbf{v}_{s}-\mathbf{v}=\mathbf{A} \dot{\mathbf{u}},
$$

where $\dot{\mathbf{u}}$ is the nodal velocity vector. With the matrix of the derivatives $\mathbf{A}^{\prime}$ e.g. a derivative $\delta \boldsymbol{\rho}_{\xi}$ of a vector $\delta \boldsymbol{\rho}$ can be defined as

$$
\delta \boldsymbol{\rho}_{\xi}=-\mathbf{A}^{\prime} \delta \mathbf{u} \text {. }
$$

We note that the matrices $\mathbf{A}$ and $\mathbf{A}^{\prime}$ are sufficient to build the tangent matrices as well as the residual vector for any arbitrary contact surface.

\subsection{Linear contact element.}

The simplest approximation is a linear contact element within the node-tosegment approach. This linear contact element has 3 nodes: the first two nodes 
$\mathbf{x}^{1}, \mathbf{x}^{2}$ are approximating a contact boundary, while the third node $\mathbf{x}^{S}$ is the slave node. The approximation on the master element is defined as

$$
\boldsymbol{\rho}(\xi):=\frac{1-\xi}{2} \mathbf{x}^{1}+\frac{1+\xi}{2} \mathbf{x}^{2}=\frac{1-\xi}{2}\left(\begin{array}{l}
x_{1} \\
y_{1}
\end{array}\right)+\frac{1+\xi}{2}\left(\begin{array}{c}
x_{2} \\
y_{2}
\end{array}\right) .
$$

The tangent vector $\boldsymbol{\rho}_{\xi}$ is then given as:

$$
\boldsymbol{\rho}_{\xi}=\frac{\mathbf{x}^{2}-\mathbf{x}^{1}}{2}=\frac{1}{2}\left(\begin{array}{c}
x_{2}-x_{1} \\
y_{2}-y_{1}
\end{array}\right) .
$$

A single metric coefficient becomes:

$$
a_{11}=\boldsymbol{\rho}_{\xi} \cdot \boldsymbol{\rho}_{\xi}=0.25 \cdot\left(\left(x_{1}-x_{2}\right)^{2}+\left(y_{1}-y_{2}\right)^{2}\right),
$$

which is the square of the length of the vector $\boldsymbol{\rho}_{\xi}$. The unit normal vector $\boldsymbol{\nu}$ to the contact segment is defined in a Cartesian coordinate system via a cross product of the tangent vector $\boldsymbol{\rho}_{\xi}$ and the third unit vector $\mathbf{k}$ which is normal to the plane:

$$
\boldsymbol{\nu}:=\frac{\left[\mathbf{k} \times \boldsymbol{\rho}_{\xi}\right]}{\left(\boldsymbol{\rho}_{\xi} \cdot \boldsymbol{\rho}_{\xi}\right)}=\frac{1}{2 a_{11}}\left(\begin{array}{c}
y_{2}-y_{1} \\
x_{1}-x_{2}
\end{array}\right) .
$$

In this definition according to eqn. (7) and to Fig. 1, we assume that the solid body occupies the lower part relative to the contact element in Fig. 3. The matrix of the shape functions $\mathbf{A}$

$$
\mathbf{A}=\left[\begin{array}{cccccc}
-\frac{1-\xi}{2} & 0 & -\frac{1+\xi}{2} & 0 & 1 & 0 \\
0 & -\frac{1-\xi}{2} & 0 & -\frac{1+\xi}{2} & 0 & 1
\end{array}\right]
$$

and the matrix of the derivatives $\mathbf{A}^{\prime}$

$$
\mathbf{A}^{\prime}=\frac{1}{2}\left[\begin{array}{cccccc}
1 & 0 & -1 & 0 & 0 & 0 \\
0 & 1 & 0 & -1 & 0 & 0
\end{array}\right]
$$

are used to approximate the displacement field as well as the derivatives. The displacement vector $\mathbf{u}$ is defined as

$$
\mathbf{u}^{T}=\left\{u_{x}^{(1)}, u_{y}^{(1)}, u_{x}^{(2)}, u_{y}^{(2)}, u_{x}^{(S)}, u_{y}^{(S)}\right\}^{T}
$$

\subsubsection{Closest point procedure.}

Consider now the increment $\Delta \xi$ in load step $(m)$ for the closest point procedure in eqn. (4) in order to define the internal coordinate $\xi_{C}$ of the projection point $C$. Due to the linear approximation in the contact element we get $\boldsymbol{\rho}_{\xi \xi}=0$. 


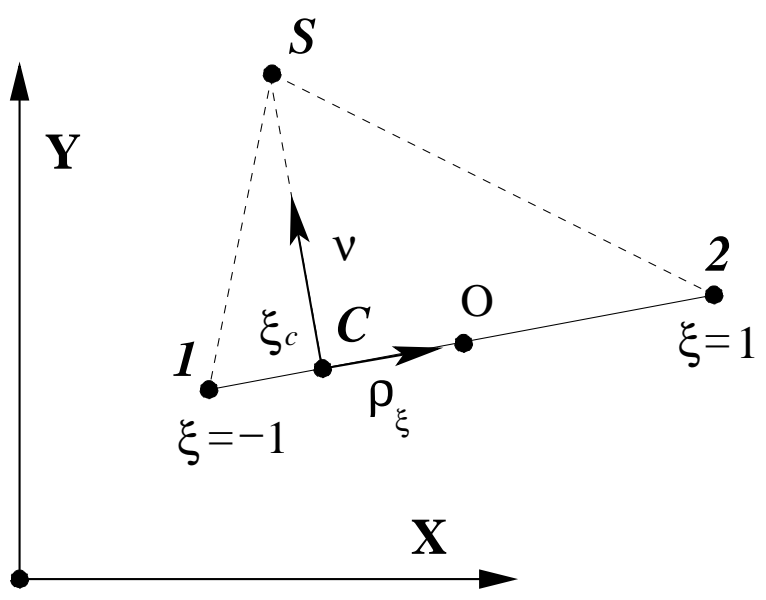

Figure 3: Linear contact element. Node-to-segment approach.

$$
\begin{aligned}
\Delta \xi=\frac{\left(\mathbf{r}_{s}-\boldsymbol{\rho}\right) \cdot \boldsymbol{\rho}_{\xi}}{\left(\boldsymbol{\rho}_{\xi} \cdot \boldsymbol{\rho}_{\xi}\right)} & =\frac{4}{\left\|\mathbf{x}^{2}-\mathbf{x}^{1}\right\|^{2}}\left(\mathbf{x}^{S}-\frac{\mathbf{x}^{1}+\mathbf{x}^{2}}{2}-\frac{\mathbf{x}^{2}-\mathbf{x}^{1}}{2} \xi^{(m)}\right) \cdot \frac{\mathbf{x}^{2}-\mathbf{x}^{1}}{2}= \\
& =\frac{2 \mathbf{x}^{S} \cdot\left(\mathbf{x}^{2}-\mathbf{x}^{1}\right)}{\left\|\mathbf{x}^{2}-\mathbf{x}^{1}\right\|^{2}}-\frac{\left\|\mathbf{x}^{2}\right\|^{2}-\left\|\mathbf{x}^{1}\right\|^{2}}{\left\|\mathbf{x}^{2}-\mathbf{x}^{1}\right\|^{2}}-\xi^{(m)} .
\end{aligned}
$$

It is obvious that the Newton update scheme $\xi^{(m+1)}=\xi^{(m)}+\Delta \xi$ in eqn. (4) is independent of the initial guess $\xi^{(m)}$, therefore, the last expression in eqn. (71) together with this scheme leads to the exact value for the internal coordinate $\xi_{C}$ :

$$
\xi_{C}=\frac{2 \mathbf{x}^{S} \cdot\left(\mathbf{x}^{2}-\mathbf{x}^{1}\right)}{\left\|\mathbf{x}^{2}-\mathbf{x}^{1}\right\|^{2}}-\frac{\left\|\mathbf{x}^{2}\right\|^{2}-\left\|\mathbf{x}^{1}\right\|^{2}}{\left\|\mathbf{x}^{2}-\mathbf{x}^{1}\right\|^{2}} .
$$

The result can be also interpreted as convergence in one iteration. Since the geometry of this element is quite simple, the same result can be also obtained directly via the triangle in Fig. 3.

A simple searching algorithm leads to computations of all further components for a contact element at the projection point $\xi_{C}$ only if the slave point $S$ is projected onto this element, i.e. if $\left|\xi_{C}\right| \leq 1$.

\subsubsection{Return-mapping scheme}

In order to define the tangent traction vector $\mathbf{T}$, we apply the standard returnmapping scheme based on the elasto-plastic analogy, as is e.g. presented in the books of Wriggers [26] and Laursen [10]. The trial tangential traction is computed via the evolution equation (36), which in the case of a linear approximation is reduced to: 


$$
\dot{T}=-\epsilon_{T}\left(\boldsymbol{\rho}_{\xi} \cdot \boldsymbol{\rho}_{\xi}\right) \dot{\xi}
$$

We consider here the simplest case for frictional problems: a quasi-statical motion leading to the development from sticking to sliding. In addition, we assume that during motion a slave point is not crossing an element boundary. Thus, for our case we need only one additional variable $\xi^{0}$ per contact element in order to define the initial position of the slave point on the master segment. The trial tangent traction is obtained via the application of the backward Euler scheme within the evolution equation (73). Since we have a linear approximation for the geometry, we have

$$
T^{(m+1)}=T^{(m)}-\epsilon_{T} a_{11}\left(\xi^{(m+1)}-\xi^{(m)}\right)=
$$

and continuing recursively, we obtain

$$
=T^{(m-1)}-\epsilon_{T} a_{11}\left(\xi^{(m+1)}-\xi^{(m-1)}\right)=\ldots=T^{(0)}-\epsilon_{T} a_{11}\left(\xi^{(m+1)}-\xi^{0}\right) .
$$

Assuming in addition, that at the initial position the tangential traction $T^{(0)}$ was zero, we obtain

$$
T_{t r}^{(m+1)}=-\epsilon_{T} a_{11}\left(\xi^{(m+1)}-\xi^{0}\right) .
$$

The last eqn. (75) serves now to compute the trial tangential reaction.

The return-mapping following the Coulomb friction condition leads then with:

$$
N^{(m+1)}=\epsilon_{N} \zeta^{(m+1)},
$$

to

$$
T^{(m+1)}= \begin{cases}T_{t r}^{(m+1)} & \text { if } \quad\left|T_{t r}^{(m+1)}\right|<\mu\left|N^{(m+1)}\right| \sqrt{a_{11}} \quad \text { (sticking) } \\ \mu\left|N^{(m+1)}\right| \sqrt{a_{11}} \operatorname{sgn}\left(T_{t r}^{(m+1)}\right) & \text { if } \quad\left|T_{t r}^{(m+1)}\right| \geq \mu\left|N^{(m+1)}\right| \sqrt{a_{11}} \quad \text { (sliding) }\end{cases}
$$

The inequality condition in eqn. (76) is obtained from the following:

$$
\left\|\mathbf{T}_{t r}^{(m+1)}\right\|<\mu|N| \quad \Longrightarrow \quad \frac{T_{t r}^{(m+1)}}{\sqrt{a_{11}}}<\mu|N|,
$$

where eqn. (23) for the absolute value of the covariant vector is taken into account.

The global solution scheme for the simplest case discussed here is presented in Table 1. 


\section{Treatment of special cases}

In this section, we consider, how to treat some particularities, which were mentioned and excluded in section 5.1.2. The first problem is arising when the applied load is not simply modified proportionally. In this situation a trial load can not be computed only via eqn. (75), because the attraction point $\xi^{0}$ must be updated. Thus we have to extend the algorithm as is shown in the following. The second problem is arising when the projection point is crossing an element boundary during the incremental loading. In this case, the computation according to eqn. (75) will produce a jump, because the convective coordinate $\xi$ belongs to different elements, see Wriggers [26] and Laursen [10].

\subsection{Update of the sliding displacements in the case of reversible loading}

We consider a geometrical interpretation of the return-mapping scheme in eqn. (76) together with the evolution equation (75), see Fig. 4.

$$
\begin{gathered}
\left|T_{t r}^{(m)}\right|<\mu\left|N^{(m)}\right| \sqrt{a_{11}} \Longrightarrow \quad \epsilon_{T}\left|\xi^{(m)}-\xi^{0}\right|<\mu\left|N^{(m)}\right| \\
\left|\xi^{(m)}-\xi^{0}\right|<R_{\xi}^{(m)}, \quad R_{\xi}^{(m)}=\frac{\mu\left|N^{(m)}\right|}{\epsilon_{T}}
\end{gathered}
$$

Eqn. (78b) describes an allowable elastic region $\mathbf{A}^{(m)} \mathbf{B}^{(m)}$ with a center of attraction $\mathbf{O}^{(m)}$. All points inside this domain are in "sticking condition". If now a point $\xi^{(m+1)}$ appears to be outside of the domain at load step $(m+1)$, then its only admissible position is on the boundary of the domain, i.e. must coincide with $\mathbf{B}^{(m+1)}$. A sliding force is applied then at the contact point, see eqn. (76). As long as we have a motion of the contact point only in one direction the sign function for the sliding force $\operatorname{sgn}\left(T_{t r}^{(m+1)}\right)=\operatorname{sgn}\left(\Delta \xi^{(m+1)}\right)$ does not change and the computation will be correct. However, when a reversible load is applied which forces the contact point to move forward or backward, the attraction point $\mathbf{O}^{(m)}$ must be updated, in order to define the sign function for the sliding force correctly. This update can be defined geometrically from Fig. 4:

$$
\left|\Delta \xi^{(m+1)}\right|=\left|\Delta \xi_{s l}\right|+R_{\xi}^{(m+1)}=\left|\Delta \xi^{(m+1)}\right|-\frac{\mu\left|N^{(m)}\right|}{\epsilon_{T}} .
$$

The absolute value of the sliding displacement is then computed at load step $(m+1)$ as:

$$
\left|\Delta \xi_{s l}\right|=\left|\xi^{(m+1)}-\xi^{(0)}\right|-\frac{\mu\left|N^{(m+1)}\right|}{\epsilon_{T}}
$$

and the updated center of the elastic domain becomes:

$$
\xi_{c}^{(u p)}=\xi^{(0)}+\operatorname{sgn}\left(\xi^{(m+1)}-\xi^{(0)}\right)\left|\Delta \xi_{s l}\right| .
$$


For the next step, the evolution equation (75) is corrected as

$$
T^{(m+2)}=-\epsilon_{T} a_{11}\left(\xi^{(m+2)}-\xi_{c}^{u p}\right) .
$$

Remark.

As an alternative procedure the back-substitution of the evolution equation (75) into eqn. (80) gives the updated scheme via the trial force:

$$
\left|\Delta \xi_{s l}\right|=\frac{1}{\epsilon_{T}}\left(\left|T_{t r}^{(m+1)}\right|-\mu \mid N^{(m+1) \mid}\right),
$$

which can be found e.g. in Wriggers [26].

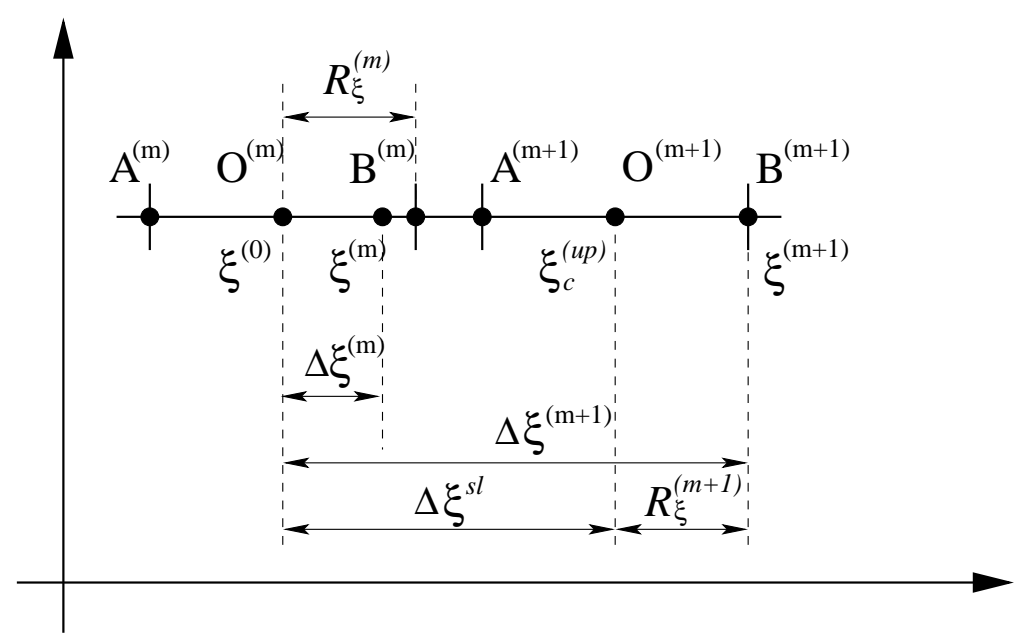

Figure 4: Coulomb friction. Updating of sliding displacements in convective coordinates. Motion of friction cone and center of attraction.

\subsection{Crossing an element boundary - continuous integration scheme}

Consider two adjacent elements $\mathbf{I}^{(m)} \mathbf{J}^{(m)}$ and $\mathbf{J}^{(m)} \mathbf{L}^{(m)}$ at load step $(m)$, see Fig. 5. Let the contact point $\mathbf{S}^{(m)}$ be projected onto the element $\mathbf{I}^{(m)} \mathbf{J}^{(m)}$ and its projection is a point $\mathbf{K}^{(m)}$ with the convective coordinate $\xi^{(m)}$. At load step $(m+1)$ these two elements move into the position $\mathbf{I}^{(m+1)} \mathbf{J}^{(m+1)}$ and $\mathbf{J}^{(m+1)} \mathbf{L}^{(m+1)}$, but the contact point is moved into position $\mathbf{S}^{(m+1)}$ and is projected now onto element $\mathbf{J}^{(m+1)} \mathbf{L}^{(m+1)}$. We assume that the angular deformation of these elements is small in comparison with a rigid body motion, thus the elements are drawn as straight lines. It is obvious, that the direct computation according to the evolution equation (75) results in a jump. Let e.g. the point $\mathbf{K}^{(m)}$ have the convective coordinate $\xi^{(m)}=0.9$ close to the right element boundary, (see parameterization in Fig. 3), and then point $\mathbf{K}^{(m+1)}$ has $\xi^{(m+1)}=-0.9$ close to the left element boundary. The distance between them is only $\Delta \xi=0.2$, but the evolution equation gives:

$$
T_{t r}^{(m+1)}=-\epsilon_{T} a_{11}\left(\xi^{(m+1)}-\xi^{(m)}\right)=1.8 \epsilon_{T} .
$$


The maximum possible jump following this straightforward action is easily determined from the limit values of the convective coordinates:

$$
T_{\text {jump }}=-\epsilon_{T} a_{11}\left(\lim _{\xi \rightarrow-1+0}(\xi)_{\xi \in \mathbf{I J}}-\lim _{\xi \rightarrow+1-0}(\xi)_{\xi \in \mathbf{J L}}\right)=2 \epsilon_{T} a_{11} .
$$

This jump appears only due to the different approximation of the adjacent elements. In order to overcome this, we can compute the force in geometrical form. The incremental tangential displacements $\Delta \rho$ can be expressed in the metrics of the second element $\mathbf{J}^{(m+1)} \mathbf{L}^{(m+1)}$ at time step $(m+1)$ :

$$
\Delta \boldsymbol{\rho}=\Delta \xi \boldsymbol{\rho}_{\xi}^{(m+1)},
$$

and alternatively, it can be geometrically defined via the incremental displacement vector $\Delta \mathbf{u}$ :

$$
\Delta \boldsymbol{\rho}=\boldsymbol{\rho}\left(\xi^{(m+1)}\right)_{\xi \in \mathbf{J L}}-\left(\boldsymbol{\rho}\left(\xi^{(m)}\right)+\Delta \mathbf{u}\left(\xi^{(m)}\right)\right)_{\xi \in \mathbf{I J}} .
$$

Then $\Delta \xi$ is defined as

$$
\Delta \xi=\frac{\left(\boldsymbol{\rho}\left(\xi^{(m+1)}\right)_{\xi \in \mathbf{J L}}-\left(\boldsymbol{\rho}\left(\xi^{(m)}\right)+\Delta \mathbf{u}\left(\xi^{(m)}\right)\right)_{\xi \in \mathbf{I J}}\right) \cdot \boldsymbol{\rho}_{\xi}^{(m+1)}}{a_{11}^{(m+1)}}
$$

and the evolution equation becomes

$$
T_{t r}^{(m+1)}=T^{(m)}-\epsilon_{T} a_{11}^{(m+1)} \Delta \xi .
$$

Modifications of the global solution scheme given in Table 1 are represented in Table 2 according to the special cases.

\subsection{Remarks on additional developments}

One can see, that the continuous integration scheme as presented in Section 6.2 leads to an increasing number of history variables, in fact, in addition to $\xi^{(m)}$ the vector $\boldsymbol{\rho}\left(\xi^{(m)}\right)$ must be stored. Moreover, other history variables such as the updated sliding displacements must be transfered in a similar fashion. The continuous integration scheme, of course, is particularly important for contact problems with singularities, e.g. sliding of an edge along a curve as also shown in Fig. 5. For other cases with nonsingular geometry it is more efficient to exclude contact points, once they appear outside the master element, but then, as a compensation, introduce additional contact points in the slave segment, e.g. integration points, within the so-called segment-to-segment approach, for details see Zavarise and Wriggers [27] especially for the 2D case and Harnau, Konyukhov and Schweizerhof [2] for the 3D case. Another approach to increase 


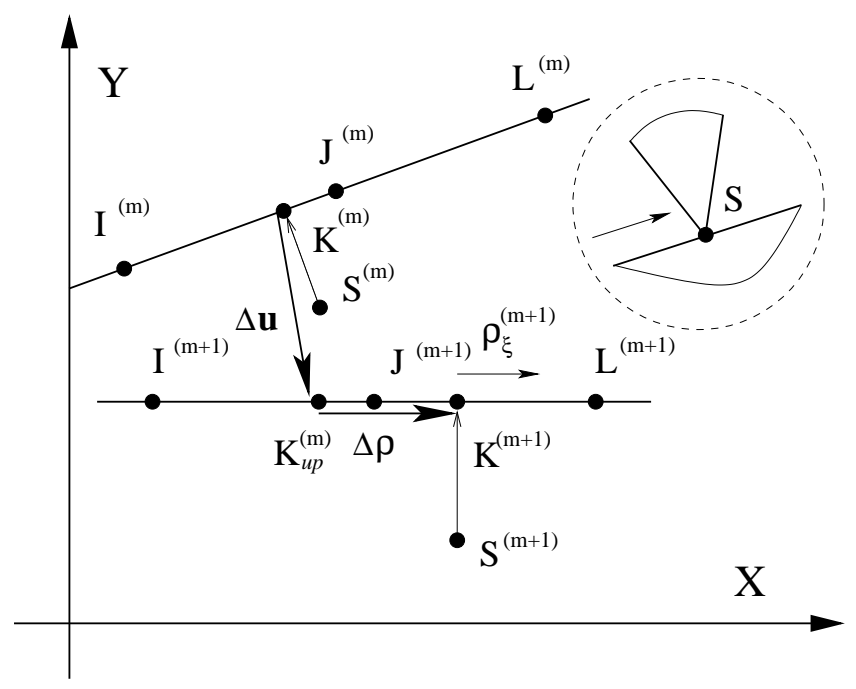

Figure 5: Crossing an element boundary within a load increment. Typical case for the continuous integration scheme.

the number of contact points is the mortar method, see e.g. in McDevitt and Laursen [12] and recent developments in Puso and Laursen [18].

Another important problem arises due to the non-smoothness of contact boundaries, if for the boundary a low order finite element mesh is used. This leads to jumps in both the normal and the tangential characteristics when crossing the element boundary see Fig. 6 , because neither a normal vector $\mathbf{n}$, nor a tangent vector $\boldsymbol{\rho}_{\xi}$ are defined at the edge point $\mathbf{B}$. If the real boundary is an edge then e.g. adaptive methods can improve the result for a straight geometry, see Wriggers and Scherf [25] and Wriggers [26]. If the real boundary is smooth, then various smoothing techniques based on the approximation of the boundary with e.g. splines can be used. There are numerous publications on this subject, see Wriggers et. al. [22], Padmanabhan and Laursen [13] and Stadler et.al. [19] especially for 2D problems, and then in Puso and Laursen [17], Krstulovic-Opara et. al. [8], Stadler and Holzapfel [20] for 3D problems. In this case, the geometrical singularity is removed, i.e. the normal $\mathbf{n}$ and the tangent vector $\boldsymbol{\rho}_{\xi}$ are uniquely defined at point B. However, the continuous integration scheme in eqn. (88-89) is still necessary, as the smooth patches have in all above publications local support, i.e. their convective coordinate is defined separately. 


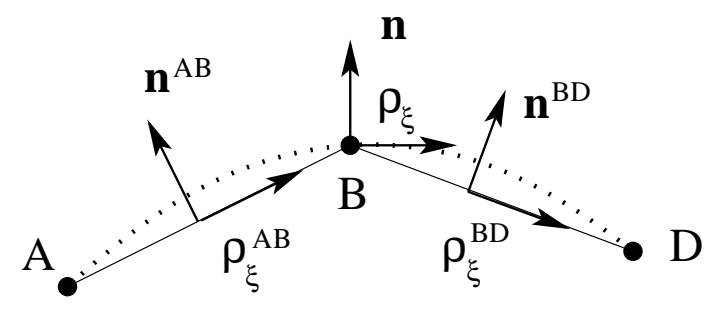

Figure 6: Low-order finite element approximation. Approximation of a real boundary with a smooth curve. 
Table 1: Global solution scheme for a linear contact element.

1. Initialization of the convective coordinate $\xi^{0}$.

Initial condition for the evolution equation for all contact elements:

Compute projection point $\xi_{C}$ in eqn. (72), with no external loads $\longrightarrow \xi^{0}=\xi_{C}$.

2. Loop over load increments $m, m=1, \ldots, m_{\text {end }}$.

3. Loop over global Newton iterations $i, i=1, \ldots, i_{\text {end }}$.

4. Loop over all contact elements

- compute projection points $\xi_{C}^{(i)}$ eqn. (72). If $\left|\xi_{C}^{(i)}\right| \geq 1$ then exit loop 4.

- check penetration $\zeta^{(i)}=\left(\mathbf{r}_{s}-\boldsymbol{\rho}\right) \cdot \boldsymbol{\nu}$. If $\zeta^{(i)}>0$ then exit loop 4.

- compute contact tractions and corresponding tangent matrices at contact point $\xi_{C}$.

Normal traction: $N^{(i)}=\epsilon_{N} \zeta^{(i)}$

Tangent matrix $\mathbf{K}^{\mathbf{N}}$ for normal traction

$$
\mathbf{K}^{\mathbf{N}}=\epsilon_{N} \quad \mathbf{A}^{T}(\boldsymbol{\nu} \otimes \boldsymbol{\nu}) \mathbf{A}+\frac{\epsilon_{N} \zeta^{(i)}}{\left(\boldsymbol{\rho}_{\xi} \cdot \boldsymbol{\rho}_{\xi}\right)}\left(\mathbf{A}^{\prime T}\left(\boldsymbol{\nu} \otimes \boldsymbol{\rho}_{\xi}\right) \mathbf{A}+\mathbf{A}^{T}\left(\boldsymbol{\rho}_{\xi} \otimes \boldsymbol{\nu}\right) \mathbf{A}^{\prime}\right)
$$

Trial tangent traction: $T_{t r}^{(i)}=-\epsilon_{T}\left(\boldsymbol{\rho}_{\xi} \cdot \boldsymbol{\rho}_{\xi}\right)\left(\xi_{C}^{(i)}-\xi^{0}\right)$

Real tangent traction $T$ and corresponding matrices are defined via the return-mapping algorithm
if $\left|T_{t r}^{(i)}\right| \leq \mu\left|N^{(i)}\right||| \boldsymbol{\rho}_{\xi} \|$
if $\left|T_{t r}^{(i)}\right|>\mu\left|N^{(i)}\right| \mid \boldsymbol{\rho}_{\xi} \|$

sticking

sliding

$T^{(i)}=T_{t r}$

Tangent matrix $\mathbf{K}^{\mathbf{T}}$

$$
\begin{aligned}
\mathbf{K}^{\mathbf{T}} & =-\frac{\varepsilon_{T}}{\left(\boldsymbol{\rho}_{\xi} \cdot \boldsymbol{\rho}_{\xi}\right)} \mathbf{A}^{T}\left(\boldsymbol{\rho}_{\xi} \otimes \boldsymbol{\rho}_{\xi}\right) \mathbf{A}+ \\
& +\frac{\varepsilon_{T}}{\left(\boldsymbol{\rho}_{\xi} \cdot \boldsymbol{\rho}_{\xi}\right)^{2}}\left[\mathbf{A}^{\prime T}\left(\boldsymbol{\rho}_{\xi} \otimes \boldsymbol{\rho}_{\xi}\right) \mathbf{A}+\mathbf{A}^{T}\left(\boldsymbol{\rho}_{\xi} \otimes \boldsymbol{\rho}_{\xi}\right) \mathbf{A}^{\prime T}\right]
\end{aligned}
$$

$T^{(i)}=\mu\left|N^{(i)}\right|\left\|\boldsymbol{\rho}_{\xi}\right\|$

Tangent matrix $\mathbf{K}^{\mathbf{T}}$

$\mathbf{K}^{\mathbf{T}}=-\frac{\varepsilon_{N} \mu \operatorname{sgn}\left(T_{t r}^{(i)}\right)}{\left(\boldsymbol{\rho}_{\xi} \cdot \boldsymbol{\rho}_{\xi}\right)^{1 / 2}} \mathbf{A}^{T}\left(\boldsymbol{\rho}_{\xi} \otimes \boldsymbol{\nu}\right) \mathbf{A}+$

$+\frac{\mu|N| \operatorname{sgn}\left(T_{t r}^{(i)}\right)}{\left(\boldsymbol{\rho}_{\xi} \cdot \boldsymbol{\rho}_{\xi}\right)^{3 / 2}}\left[\mathbf{A}^{\prime T}\left(\boldsymbol{\rho}_{\xi} \otimes \boldsymbol{\rho}_{\xi}\right) \mathbf{A}+\right.$

$\left.+\mathbf{A}^{T}\left(\boldsymbol{\rho}_{\xi} \otimes \boldsymbol{\rho}_{\xi}\right) \mathbf{A}^{\prime T}\right]$

- Compute the full contact tangent matrix $\mathbf{K}=\mathbf{K}^{\mathbf{N}}+\mathbf{K}^{\mathbf{T}}$

- Compute residual $\mathbf{R}$

$$
\begin{array}{ll}
\mathbf{R}_{N}=N^{(i)} \mathbf{A}^{T} \boldsymbol{\nu} ; & \mathbf{R}_{T}=\frac{T^{(i)}}{\left(\boldsymbol{\rho}_{\xi} \cdot \boldsymbol{\rho}_{\xi}\right)} \mathbf{A}^{\prime T} \boldsymbol{\rho}_{\xi}, \\
\mathbf{R}=\mathbf{R}_{N}+\mathbf{R}_{T} . &
\end{array}
$$

end loop over contact elements end loop over global Newton iterations end loop over load increments 
Table 2: Modifications of the global solution scheme according to special cases.

1. Initialization of the convective coordinate $\xi^{0}$.

Initial condition for the evolution equation for all contact elements:

Compute projection point $\xi_{C}$ in eqn. (72), with no external loads $\longrightarrow \xi^{0}=\xi_{C}$.

2. Loop over load increments $m, m=1, \ldots, m_{\text {end }}$.

3. Loop over global Newton iterations $i, i=1, \ldots, i_{\text {end }}$.

4. Loop over all contact elements

- compute projection points $\xi_{C}^{(i)}$ eqn. (72). If $\left|\xi_{C}^{(i)}\right| \geq 1$ then exit loop 4.

- check penetration $\zeta^{(i)}=\left(\mathbf{r}_{s}-\boldsymbol{\rho}\right) \cdot \boldsymbol{\nu}$. If $\zeta^{(i)}>0$ then exit loop 4.

- compute contact tractions and corresponding tangent matrices at contact point $\xi_{C}$.

Compute normal traction: $N^{(i)}$ and corr. matrix, see Table 1

Trial tangent traction $T_{t r}^{(i)}$ according to the specific algorithm:

\begin{tabular}{|l|l|}
\hline a) reversible loading & b) continuous integration \\
$T^{(i)}=-\epsilon_{T} a_{11}\left(\xi^{(i)}-\xi_{c}^{u p}\right)$. & $\Delta \xi$ see eqn. (88) \\
$T_{t r}^{(i)}=T^{(m-1)}-\epsilon_{T} a_{11}^{(i)} \Delta \xi$ \\
\hline
\end{tabular}

- Compute real tangent traction $T^{(i)}$ and corr. tangent matrices $\mathbf{K}^{\mathbf{T}}$ according to the return mapping scheme, see Table 1

- Compute the full contact tangent matrix $\mathbf{K}=\mathbf{K}^{\mathbf{N}}+\mathbf{K}^{\mathbf{T}}$, see Table 1

- Compute residual $\mathbf{R}$, see Table 1

end loop over contact elements

end loop over global Newton iterations

Update and store necessary history variables

\begin{tabular}{|l|l|}
\hline a) reversible loading & b) continuous integration \\
$\begin{array}{l}\text { Compute and store the update center } \xi_{c}^{(u p)} \\
\text { according to eqn. }(81)\end{array}$ & $\begin{array}{l}\text { Store history variables for } \Delta \xi \text { according to } \\
\text { eqn. }(88) \text { and } T^{(m)} .\end{array}$ \\
\hline
\end{tabular}

end loop over load increments 


\section{$7 \quad$ Numerical examples}

7.1 Sliding of a block. Linear approximation of the contact surfaces. Reversible loading process.

We consider the sliding of an elastic block similar as shown in [7], however, here the block will be loaded with horizontally prescribed reversible displacements. The main point is to show the update procedure for sliding displacements and investigate the development of the sticking-sliding zone. All numerical investigations are performed with FEAP-MeKa [21] including the implementation of the presented algorithms.

As an example for the computation, we consider a rectangular block (Fig. 7) resting on a surface with the following parameters: elasticity modulus $E=$ $2.1 \cdot 10^{4}$, Poisson ratio $\nu=0.3$, length $a=20$, height $b=5$. The dimensions are assumed to be consistent. The block is uniformly meshed by linear finite elements: 40 elements in horizontal direction and 10 elements in vertical direction. The lower surface represents a rigid base. Coulomb friction with a coefficient $\mu=0.3$ is specified between the surfaces. The contact surface of the deformable block is assumed to be a "master", while the surface of the rigid base is a "slave" surface within the "node-to-segment" approach. The penalty parameters are chosen as $\varepsilon_{N}=\varepsilon_{T}=2.1 \cdot 10^{6}$. The loading is applied as prescribed displacements at the top side of the deformable block. This example is chosen to show the robustness of the contact algorithm and the update scheme within a covariant description, though the current results can be achieved certainly with other known techniques.

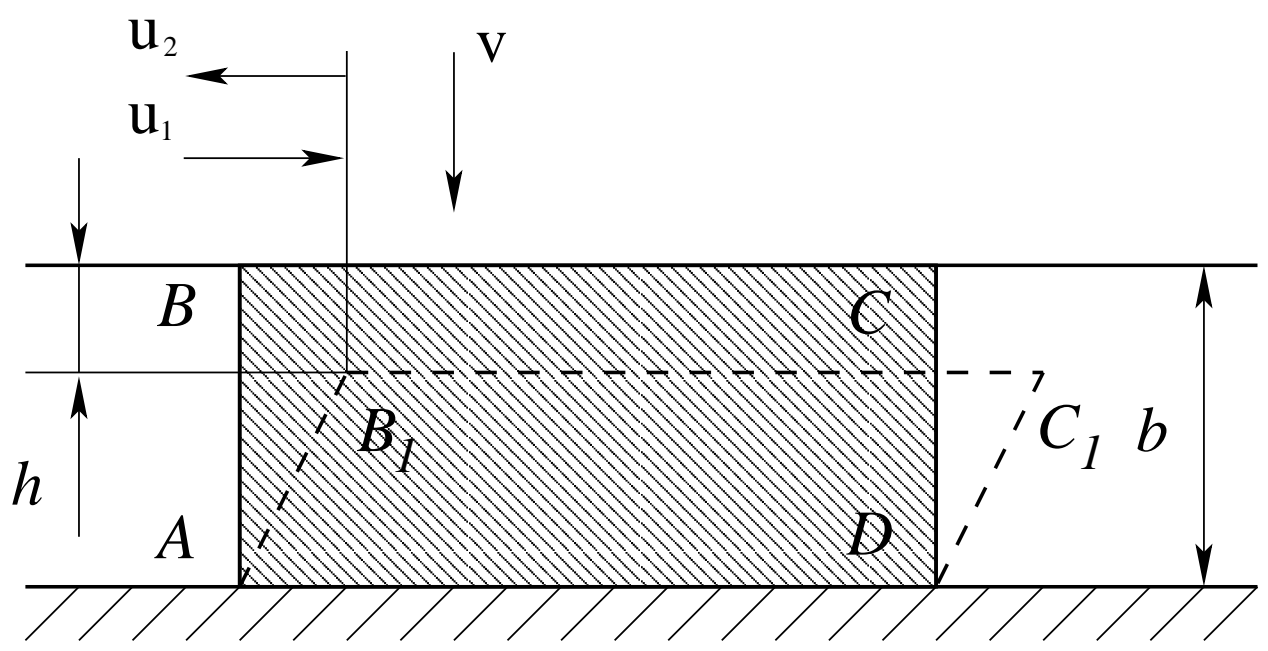

Figure 7: Plane deformation of a block. Applied displacement loading at top of the block.

The main question arising before the computation is, which size of the load step is allowed, as it is important to capture the spreading of the sticking-sliding 
zone correctly. We use the concept of the critical displacement $u_{c r}$, introduced in [7], namely, a value of the applied displacements after which the block fully slides. As shown for the infinite layer this value is given as

$$
u_{c r}=\frac{2 \mu h}{1-\nu} \text {. }
$$

The estimation of the critical horizontal displacement in eqn. (90) gives $u_{c r}=$ $6.0 \cdot 10^{-3}$, thus in order to investigate the sticking-sliding zone properly we choose a displacement increment $\Delta u=1.0 \cdot 10^{-4}$. The displacements are applied according to the loading process given in Table 3.

\begin{tabular}{|c|c|c|c|c|c|}
\hline No. l.s. & $\Delta u$ & $u \cdot 10^{-3}$ & $\Delta v \cdot 10^{-3}$ & $v$ & Loading \\
\hline 0 & 0.0 & 0.0 & 0.0 & 0 & initialization of conv. coord. \\
\hline 1 & 0.0 & 0.0 & $7.0 \cdot 10^{-3}$ & $0.0-7.0$ & vertical displ. $v$ \\
\hline $2-80$ & $10^{-4}$ & $0.0-8.0$ & 0.0 & 7.0 & forward horizontal displ. $u_{1}$ \\
\hline $81-84$ & $-2.5 \cdot 10^{-5}$ & $8.0-7.9$ & 0.0 & 7.0 & backward horizontal displ. $u_{2}$ \\
\hline $85-163$ & $-1.0 \cdot 10^{-4}$ & $7.9-0.0$ & 0.0 & 7.0 & backward horizontal displ. $u_{2}$ \\
\hline $164-280$ & $-1.0 \cdot 10^{-4}$ & $0.0-(-1.17)$ & 0.0 & 7.0 & backward horizontal displ. $u_{2}$ \\
\hline
\end{tabular}

Table 3: Plane deformation of a block. Loading procedure with prescribed displacements on the top side of the deformable block.

As a consequence of the reversible loading a hysteresis curve as shown in Fig. 8 is developed. The applied displacement at point $\mathbf{C}$, see Fig. 7, is depicted along the $x$-axis, and the computed horizontal displacement at point $\mathbf{D}$ is depicted along the $y$-axis. We obtain a spreading of the sliding zone during the forward

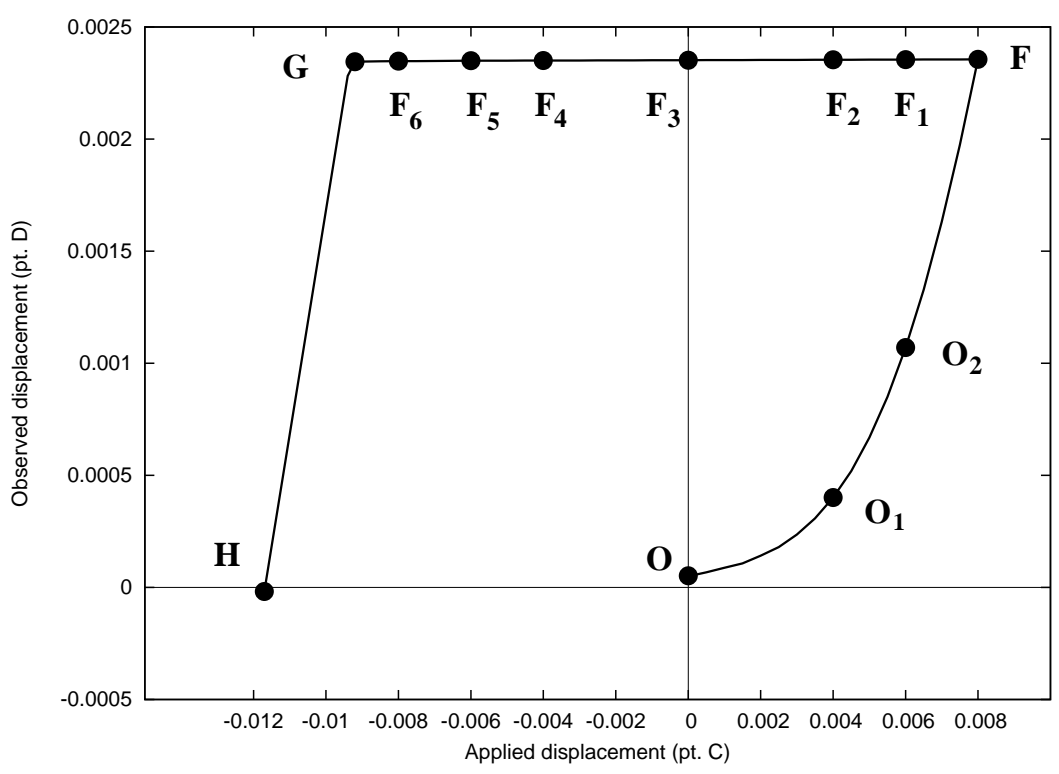

Figure 8: Plane deformation of a block. Hysteresis curve. Observed horizontal displacement at point D vs. applied horizontal displacement at point $\mathrm{C}$.

loading process (curve $\mathbf{O F}$ ) as well as during the backward loading process (curve FG). The horizontal displacements $u$ along the contact line AD, see 
Fig. 7, together with the distribution of the stress ratio $T / N$ allow us to define the sticking-sliding zone during the loading process.

Forward loading. Path OF on the hysteresis curve. The horizontal displacement distribution as well as the stress ratio $T / N$ distribution on the contact boundary are shown in Fig. 9 and Fig. 10 for the following loading points:

a) Load step 1 , see Table 3, i.e. only vertical displacements are applied $v=$ 0.007. This is the starting point $\mathbf{O}$ on the hysteresis curve Fig. 8;

b) Intermediate points with applied horizontal displacements $u=0.0040$ and $u=0.0060$, resp. points $\mathbf{O}_{1}$ and $\mathbf{O}_{2}$ on the hysteresis curve Fig. 8;

c) The load step No. 80 with $u=0.0080$, see Table 3 , is chosen as a final point of the forward loading, see also the point $\mathbf{F}$ on the hysteresis curve Fig. 8.

The development of the sliding zone with increasing displacement loading $u$ is given in Fig. 9 and the development of the stress ratio $T / N$ in Fig. 10. At the end of the forward loading the sliding zone is increased to about $8 \leq x \leq 20$.

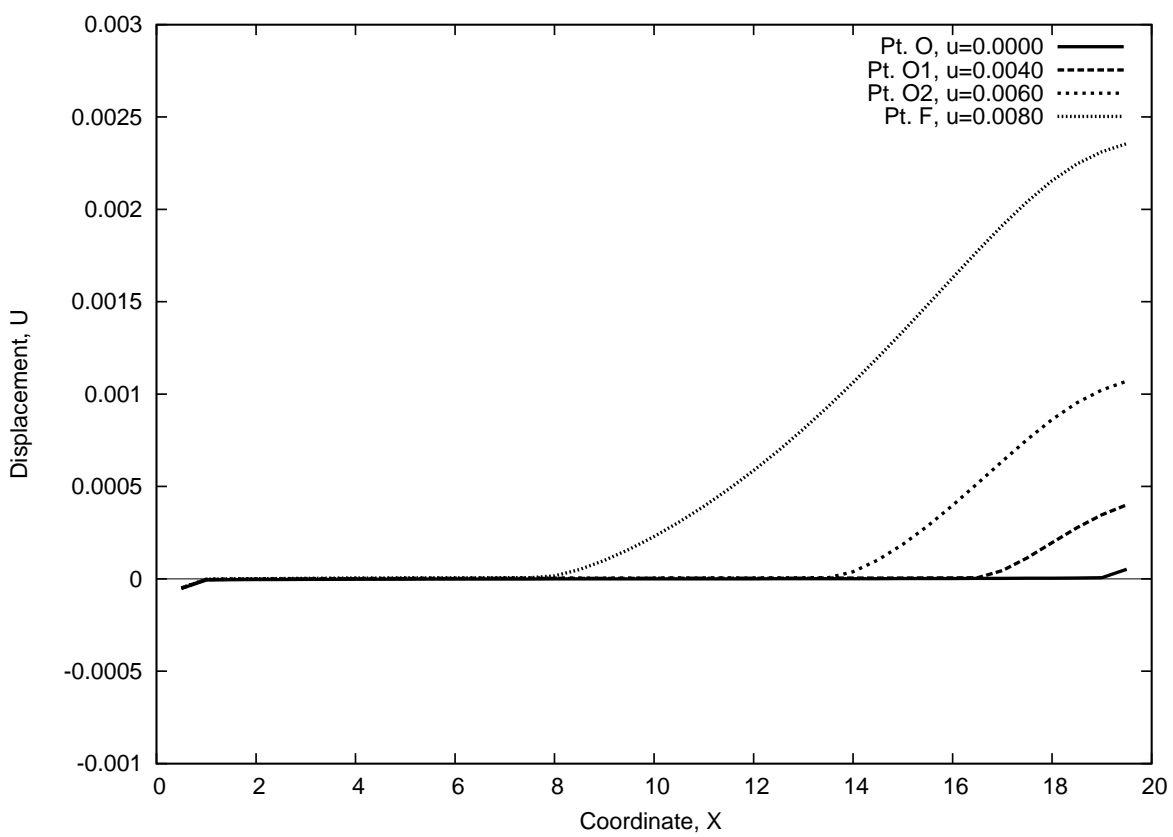

Figure 9: Plane deformation of a block. Horizontal displacement of the contact boundary. Forward loading.

Backward loading. Part FGH on the hysteresis curve. The horizontal displacement distribution and the contact stress ratio $T / N$ distribution on the contact boundary are shown in Fig. 11 and in Fig. 12 for the following loading points:

a) Last load step of the forward loading with $u=0.0080$, resp. point $\mathbf{F}$ on the hysteresis curve Fig. 8 as a starting point; 


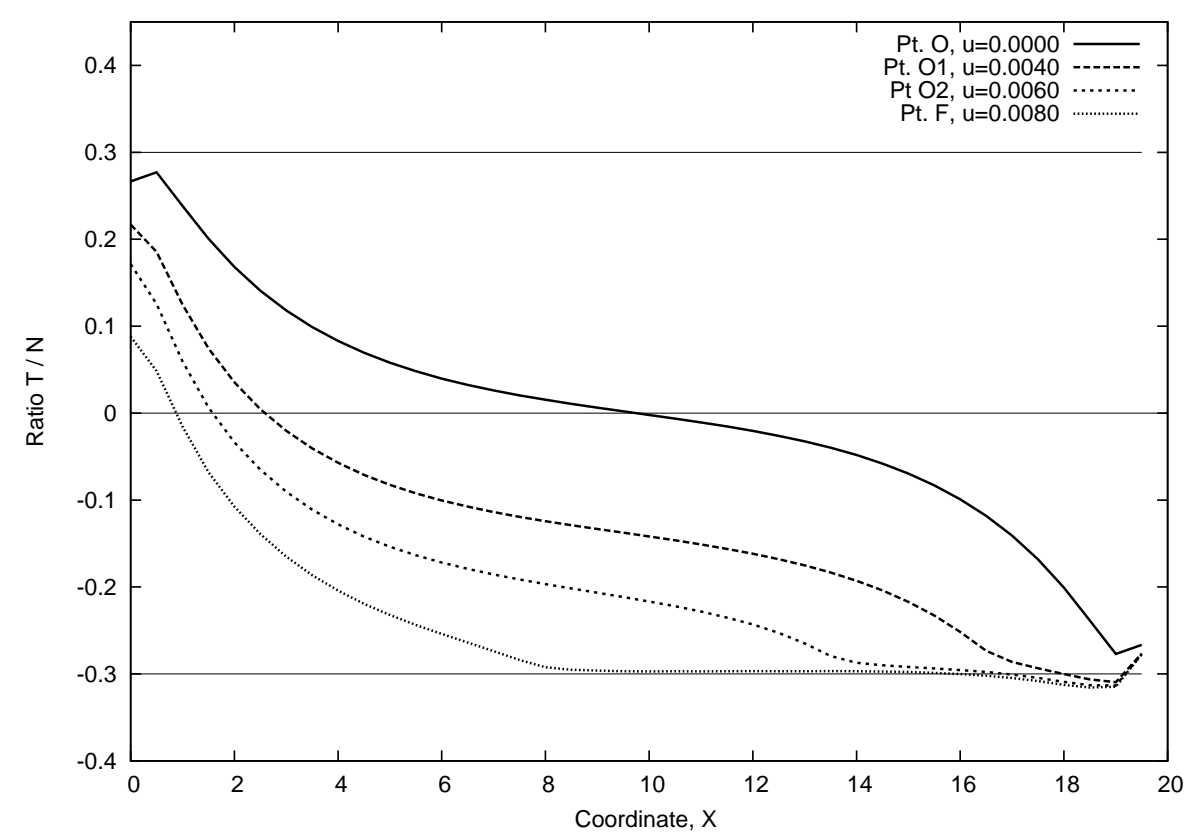

Figure 10: Plane deformation of a block. Stress ratio $T / N$ on the master contact surface. Forward loading.

b) Intermediate points on the unloading part FG with applied horizontal displacements $u=0.0040, u=0.000, u=-0.0040, u=-0.0060, u=$ -0.0080 , resp. points $\mathbf{F}_{\mathbf{2}}, \mathbf{F}_{\mathbf{3}}, \mathbf{F}_{\mathbf{4}}, \mathbf{F}_{\mathbf{5}}, \mathbf{F}_{\mathbf{6}}$ on the hysteresis curve;

c) Selected point with $u=0.010$ on the full backward sliding part $\mathbf{G H}$ of the hysteresis curve.

When the load is reversed, starting from load step No. 81, see Table 3, all points on the contact boundary are sticking. On the contact boundary we have so-called residual horizontal displacements (in analogy to plasticity). During the following unloading back to $u=0.000$ the whole boundary is still sticking: points $\mathbf{F}_{\mathbf{1}}, \mathbf{F}_{\mathbf{2}}, \mathbf{F}_{\mathbf{3}}$. The latter we can only detect from the stress ratio diagram in Fig. 12, where the curves vary inside the layer $-0.3 \leq T / N \leq 0.3$. The residual displacements are not changing, see diagram 11, until sliding is beginning. Starting from the applied displacements $u=-0.0040$ (point $\mathbf{F}_{4}$ ) we can detect the beginning of sliding at the left corner of the block, as the stress ratio curve is approaching its limit ratio 0.3 . The final part of the hysteresis diagram, from point $\mathbf{F}_{4}$ to point $\mathbf{G}$, is responsible for the development of the sliding zone in the backward direction, which can be observed either by the development of the horizontal displacements in the left part of the displacement diagram 11, or by the development of the zone with a stress ratio $T / N=0.3$ in diagram 12. Full sliding of the block starts at point $\mathbf{G}$ with $u \approx-0.0092$. Beyond that, horizontal displacements on the contact are changing proportionally to the applied displacement loading, i.e. linearly, as we see from the linear part $\mathbf{G H}$ in the 
hysteresis curve.

\section{Remark.}

In the presented example the displacements are small and slaves nodes never cross the element boundaries, therefore the continuous integration scheme is not necessary. In the next example we show a particular case with large sliding in which the application of the continuous integration scheme is necessary.

\subsection{Drawing of an elastic strip into a channel with sharp corners.}

In this section, we consider a special contact case, for which the application of the continuous integration scheme described in sect. 6.2 is absolutely necessary. An elastic strip $\mathbf{A D}$, see Fig. $13\left(E=2.1 \cdot 10^{4}, \nu=0.3\right.$, thickness $h=0.5$, length $L=24$ ) is positioned at the beginning of a channel with width $a=13$. The corners of the channel are rather sharp. The channel itself is modeled by two rigid blocks $\mathbf{B}_{\mathbf{1}}$ and $\mathbf{B}_{\mathbf{2}}$. The strip is loaded incrementally by a prescribed displacement $v$ at the center until it is inserted into the channel, see Fig. 14. The strip here is modeled with 24 linear solid-shell elements, see Hauptmann and Schweizerhof [3] and Hauptmann et.al. [4], and due to symmetry only one half of the system is modeled. The crucial point during the analysis is the sliding of a sharp corner $\mathbf{C}$ over the element boundaries 1, 2, 3, see Fig. 14 . A load-displacement curve computed for the loading point is chosen as the representative parameter to compare various contact approaches. The following variations were investigated:

1. Non-frictional case with the "node-to-surface" approach without the continuous integration scheme proposed in eqn. (88) and (89).

2. Non-frictional case with the "segment-to-segment" approach. Here the number of integration points in the contact segment is varied.

3. Frictional case with the "node-to-surface" approach without the continuous integration scheme.

4. Frictional case with the "node-to-surface" approach with the continuous integration scheme.

5. Frictional case with the "segment-to-segment" approach. Here the number of integration points in the contact segment are varied.

We start the investigation with the non-frictional problem (case 1,2) applying the load increment $v=0.005$ with the penalty parameter $\varepsilon_{N}=2.1 \cdot 10^{5}$. The elements from the strip are chosen to be a master, while the sharp corner is a 
singular slave node. The load-displacement curve for the "node-to-surface" approach contains a jump when the sharp corner is crossing the boundary nodes 1 resp. 2, see Fig. 14. The solution process is no longer converging after the sharp corner is crossing the boundary node $\mathbf{3}$. As an alternative for improvements, we chose the "segment-to-segment" approach, described in Harnau, Konyukhov and Schweizerhof [2]. The sharp corner $\mathbf{C}$ is modeled with two slave segments which are orthogonal to each other and take contact points as the Lobatto integration points. Taking 2 integration points with 2 sub-domains, or e.g. 5 integration points allows to compute the full load-displacement curve without the jumps obtained with NTS scheme, see Fig. 15. The smoothing effect in the last cases happens because the contact is checked not only against the single edge node, but also against the set of contact points which covers fairly densely the sharp edge.

The next study is devoted to the frictional problem (case 3,4 ) with the load increment $v=0.0025$, the penalty parameters $\varepsilon_{N}=2.1 \cdot 10^{5}, \varepsilon_{T}=2.1 \cdot 10^{5}$ and a friction coefficient $\mu=0.2$. The straightforward analysis without the continuous integration scheme (case 3 ) leads to a jump in the force-displacement curve when the sharp corner is crossing the first boundary node $\mathbf{1}$. The solution is no longer converging when the sharp corner is crossing the second boundary node $\mathbf{2}$, see Fig. 16. The application of the continuous integration scheme, however, allows to obtain the full force-displacement curve, even in the part when the strip is fully inserted into the channel, see the straight part of the curves in Fig. 16. For this case a side part of the channel is modeled as a rigid surface described by an analytical function. For comparison, the analysis is carried out with various friction coefficients $\mu=0.1$ and $\mu=0.3$, see Fig. 16 .

It would be favorable also for the frictional case to perform the analysis without the continuous integration scheme avoiding to store a lot of information about slave nodes and apply the "segment-to-segment" approach just increasing the number of integration points (case 2). The results of such an analysis using the same loading parameters as for the analysis in Fig. 16 are shown in Fig. 17. 5 and 10 Lobatto integration points are taken. Despite the fact that the solution is converging, the load-displacement curve shows large oscillations in the sliding regions after passing node $\mathbf{2}$. This result is due to the fact, that from one load step to the other the history variables are not transported correctly. Only the upper envelope of the oscillatory curve could be used as representation of the correct load-displacement curve. This confirms the necessity of the continuous integration scheme in particular for frictional contact.

Remark. We have to note that reversing the master surface, or the socalled symmetric treatment of the contact, in the current example would also 


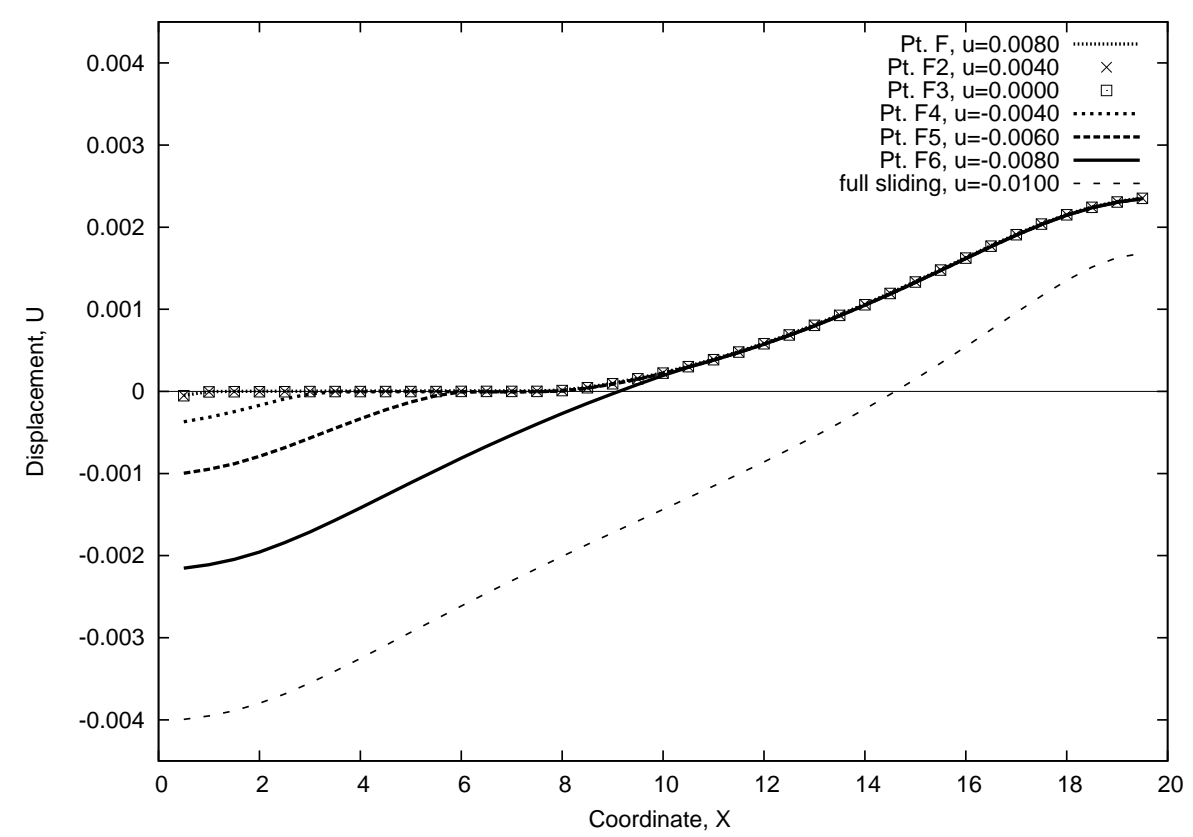

Figure 11: Plane deformation of a block. Backward loading. Horizontal displacement of the contact boundary.

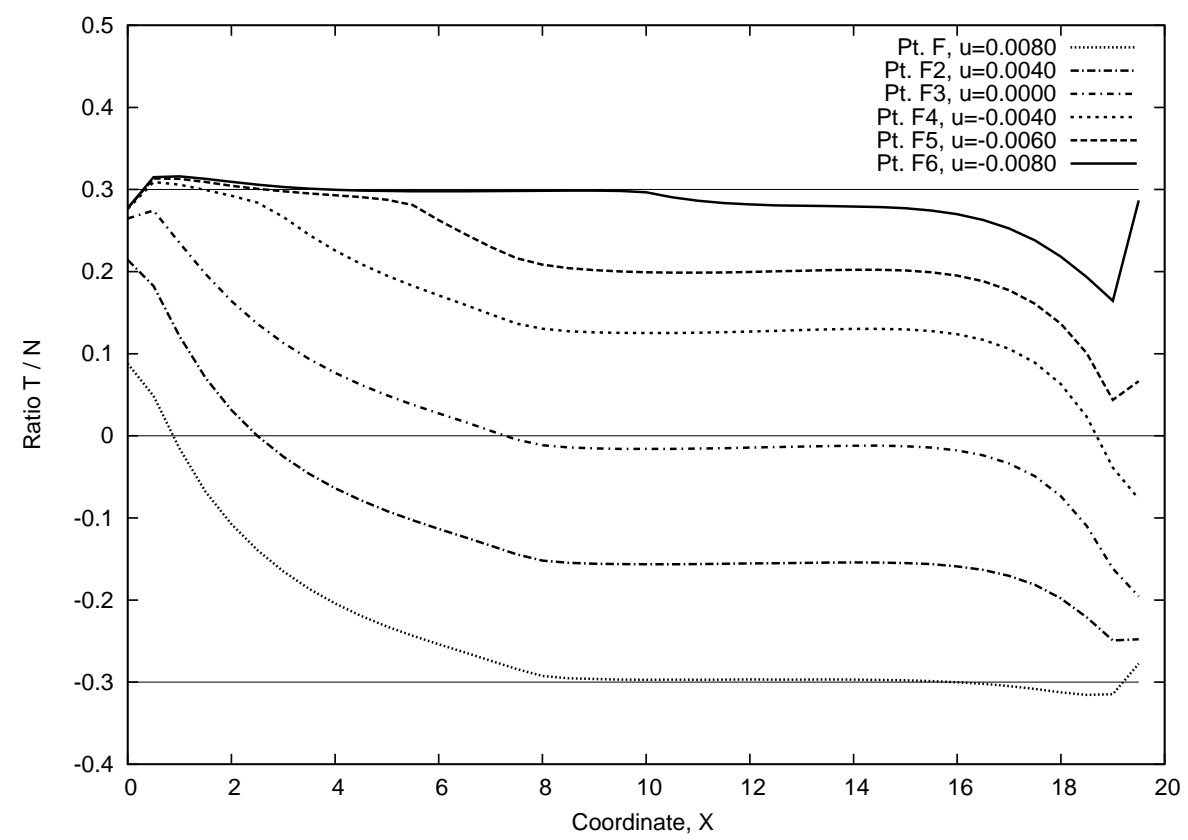

Figure 12: Plane deformation of a block. Backward loading. Stress ratio $T / N$ on the master contact surface.

not resolve the problem in a sufficient manner.

\section{Conclusions}

In this contribution a convective description was reconsidered for the 2D quasistatical frictional contact problem. Special attention is paid to the derivation of the necessary equations either as a reduction of the known 3D covariant for- 


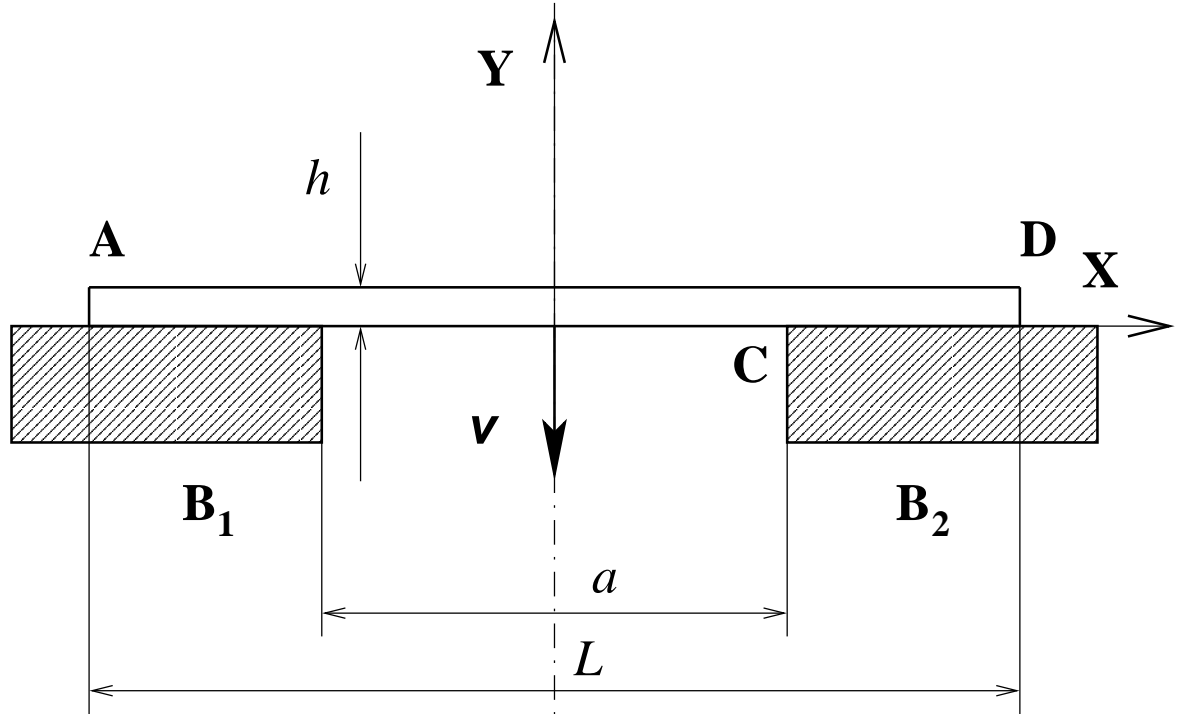

Figure 13: Drawing of an elastic strip into a channel with sharp corners. Geometrical parameters.
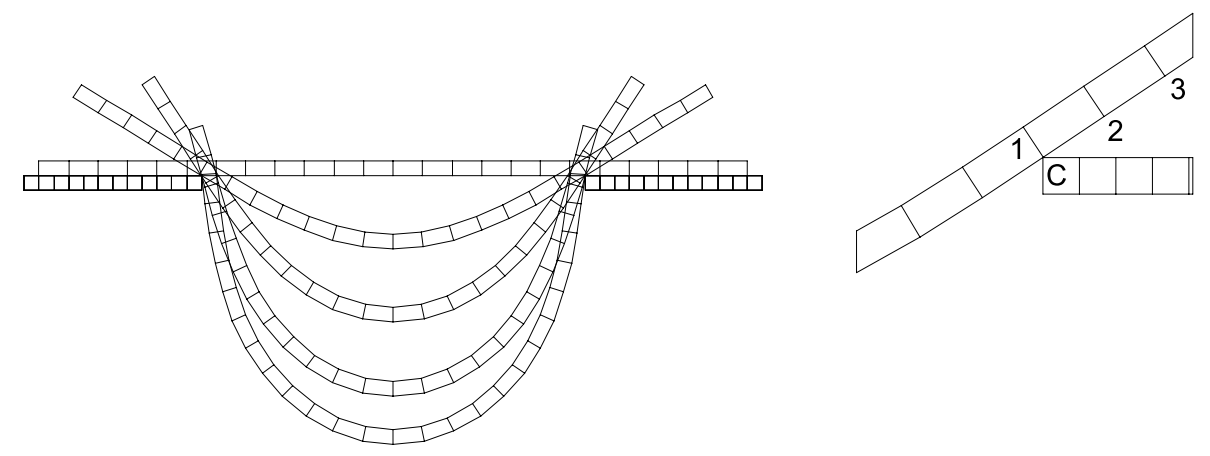

Figure 14: Sequence of deformations for the elastic strip. Nodes are sliding over the sharp corner C.

mulation, or directly from the special 2D cylindrical geometry of the contact surfaces. The algorithmic linearization in the covariant form allows to obtain the tangent matrices before the linearization process. Thus, an implementation can be easily carried out without providing any special attention to the approximation of the contact surfaces (e.g. finite element mesh, CAD surface etc.). A simple linear contact element is chosen to illustrate the algorithmic implementation into a FE code.

Different situations requiring the application of more advanced techniques, such as an update of sliding displacements and a continuous integration scheme for the frictional case are discussed and illustrated with numerical examples. Thus, the update technique is absolutely necessary for the simulation of reversible loading processes, as the residual deformations have to be described correctly. The continuous integration technique allows to transport all history 


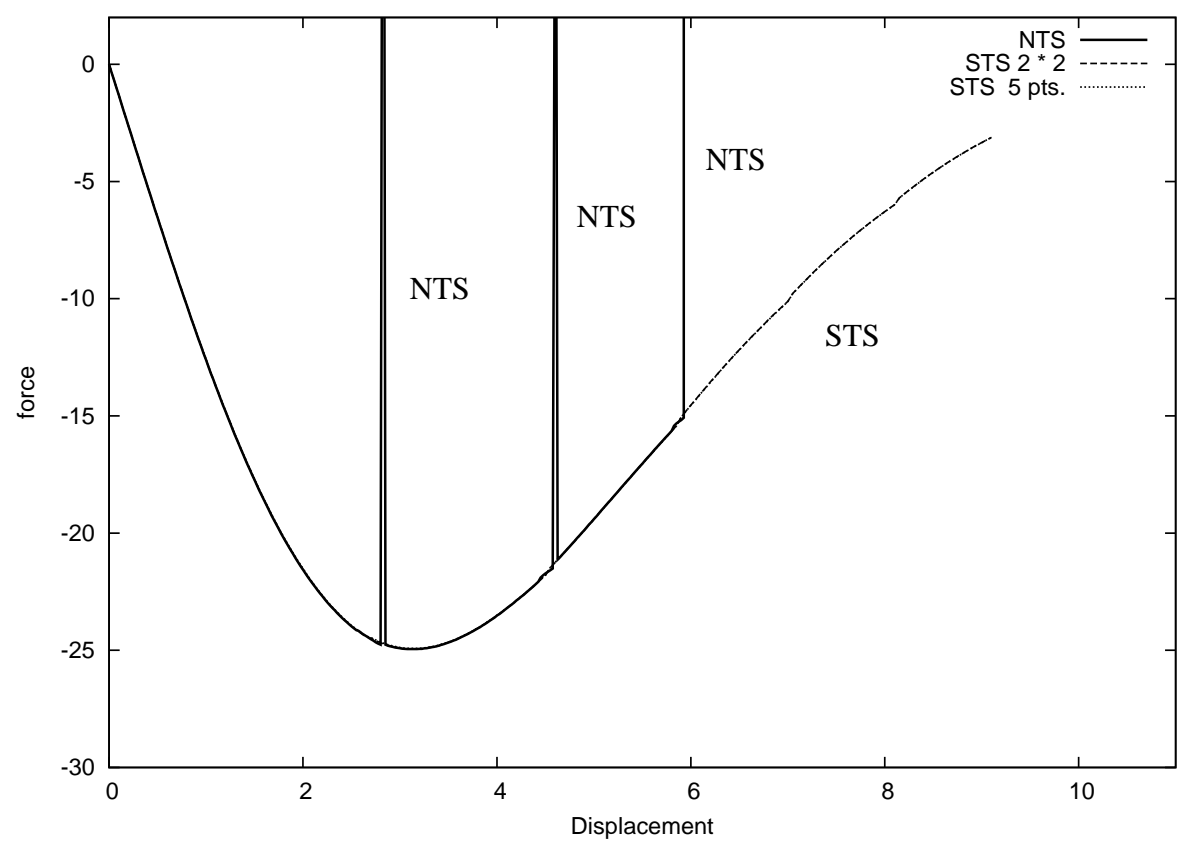

Figure 15: Drawing of an elastic strip over a sharp corner. Load-displacement curve. Non-frictional case. NTS and STS approaches with different Lobatto integration - a) $2 \times 2$ int. points with 2 sub-domains and b) $5 \times 5$ int. points.

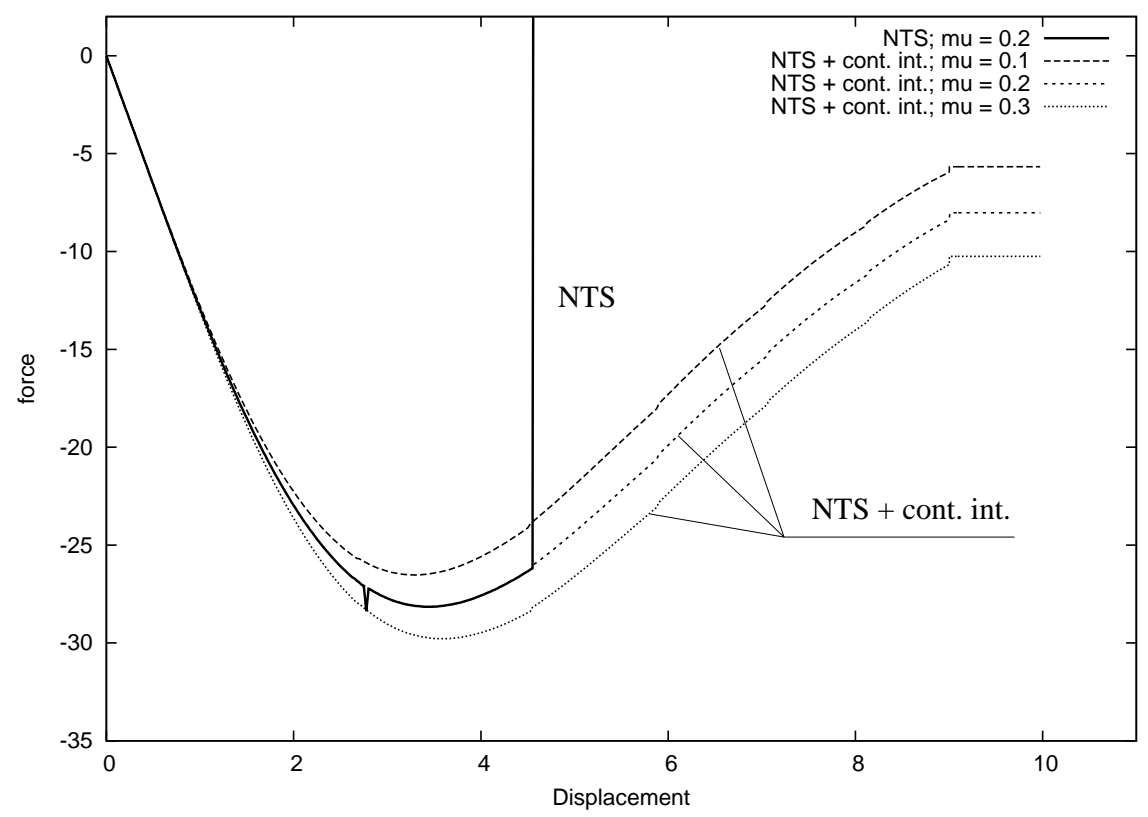

Figure 16: Drawing of an elastic strip over a sharp corner. Load-displacement curve. Frictional case - various friction coefficients. Straightforward NTS approach and continuous integration schemes.

variables correctly over the contact segment boundaries, however, additional storage is required. In the particular example of a sliding edge on a surface, it is shown that for the non-frictional contact problem the "segment-to-segment" approach with different integration schemes can improve the result, but for 


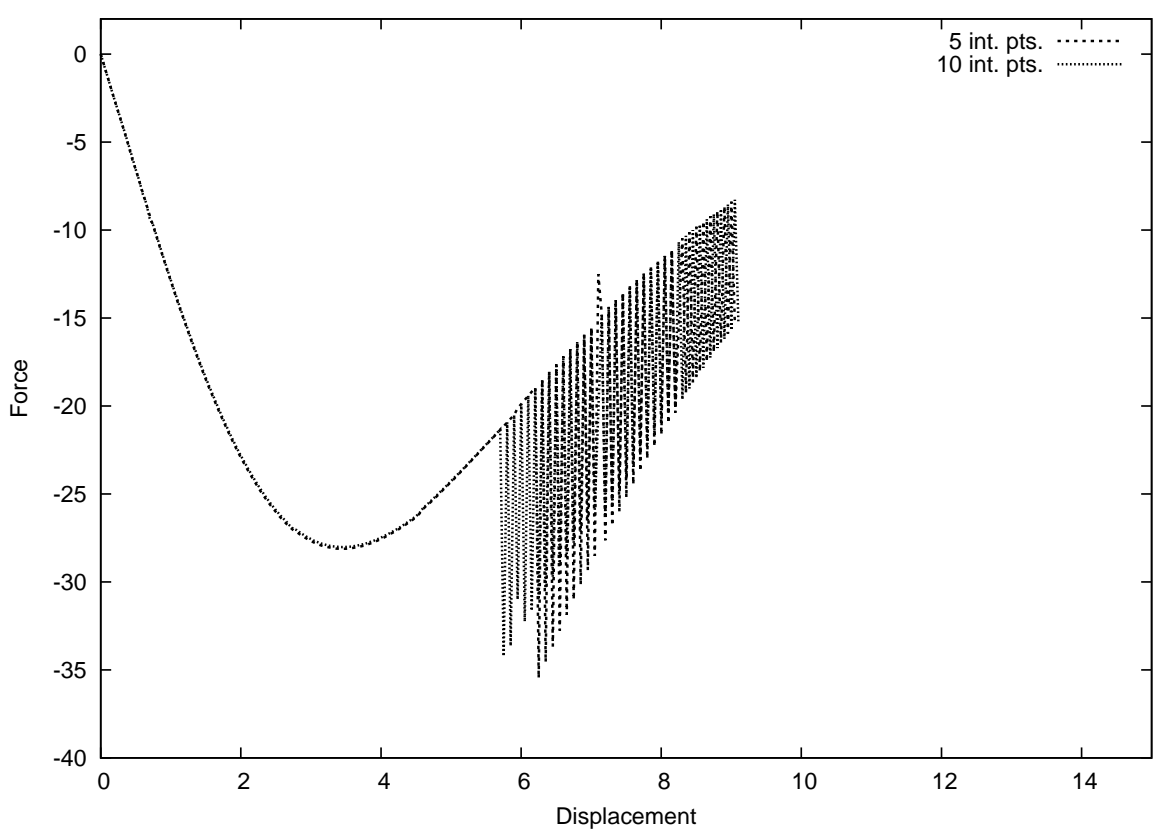

Figure 17: Drawing of an elastic strip over a sharp corner. Load-displacement curve. Frictional case. Straightforward application of the "segment-to-segment" scheme without the continuous integration scheme leads to high oscillations also for a larger number of integration points.

the frictional contact problem the continuous integration scheme is absolutely necessary independent of the approaches NTS and STS.

\section{APPENDIX. Covariant derivative of tangent vector $\mathrm{T}$}

The full time derivative of the tangent vector $\mathbf{T}$ is considered in the contravariant basis $\mathbf{r}^{1}, \mathbf{r}^{2}$

$$
\frac{d \mathbf{T}}{d t}=\frac{d}{d t}\left(T \mathbf{r}^{1}\right)=\frac{d T}{d t} \mathbf{r}^{1}+T \frac{d \mathbf{r}^{1}}{d t}
$$

For the computation we assume, that the component $T$ is a scalar function of $t, \xi$, i.e. $T=T(t, \xi)$, and the basis vector $\mathbf{r}^{1}$ depends implicitly on time via the convective coordinates $\xi$ and $\zeta$, see the definition in eqn. (17). Then we obtain:

$$
\frac{d \mathbf{T}}{d t}=\left(\frac{\partial T}{\partial t}+\frac{\partial T}{\partial \xi} \dot{\xi}\right) \mathbf{r}^{1}+T\left(\dot{\xi} \frac{\partial \mathbf{r}^{1}}{\partial \xi}+\dot{\zeta} \frac{\partial \mathbf{r}^{1}}{\partial \zeta}\right) .
$$

The partial derivatives of the contravariant basis vectors are expressed via the Christoffel symbols $\Gamma_{i j}^{k}$, see e.g. Marsden and Hughes [11],

$$
\begin{aligned}
& \frac{\partial \mathbf{r}^{1}}{\partial \xi}=\Gamma_{11}^{1} \mathbf{r}^{1}+\Gamma_{12}^{1} \mathbf{r}^{2}, \\
& \frac{\partial \mathbf{r}^{1}}{\partial \zeta}=\Gamma_{21}^{1} \mathbf{r}^{1}+\Gamma_{22}^{1} \mathbf{r}^{2} .
\end{aligned}
$$


Using the orthogonality of the covariant and contravariant basis vectors $\mathbf{r}_{i}$ and $\mathbf{r}^{i}$, we can write the following:

$$
\frac{d \mathbf{T}}{d t}=\left(\frac{d \mathbf{T}}{d t} \cdot \mathbf{r}_{1}\right) \mathbf{r}^{1}+\left(\frac{d \mathbf{T}}{d t} \cdot \mathbf{r}_{2}\right) \mathbf{r}^{2}
$$

For the formulation of the evolution equations, it is necessary to obtain only a covariant component of the full time derivative on the tangent line, therefore, from the expansion (94) we need only the first term computed at $\zeta=0$. We introduce this derivative as follows

$$
\frac{D_{1} T}{d t} \equiv\left(\frac{d \mathbf{T}}{d t} \cdot \mathbf{r}_{1}\right)_{\zeta=0}=\left(\frac{d \mathbf{T}}{d t} \cdot \boldsymbol{\rho}_{\xi}\right)=\frac{\partial T}{\partial t}+\frac{\partial T}{\partial \xi} \dot{\xi}+T\left(\Gamma_{11}^{1} \dot{\xi}+\Gamma_{21}^{1} \dot{\zeta}\right)_{\zeta=0} .
$$

One can recognize in eqn. (95) the full time derivative via the covariant derivatives. We compute the value of the necessary Christoffel symbols in brackets, according to their definition in eqn. (93) and to the definition of $\mathbf{r}_{1}$ in (17).

$$
\begin{aligned}
\left(\Gamma_{11}^{1}\right)_{\zeta=0} & =\left(\frac{\partial \mathbf{r}^{1}}{\partial \xi} \cdot \mathbf{r}_{1}\right)_{\zeta=0}, \\
\left(\Gamma_{21}^{1}\right)_{\zeta=0} & =\left(\frac{\partial \mathbf{r}^{1}}{\partial \zeta} \cdot \mathbf{r}_{1}\right)_{\zeta=0} .
\end{aligned}
$$

In order to avoid the derivative of the contravariant vector in (96), we take a derivative of the following identity

$$
\begin{gathered}
\mathbf{r}^{1} \cdot \mathbf{r}_{1}=1 \Longrightarrow \frac{\partial}{\partial(\ldots)}\left(\mathbf{r}^{1} \cdot \mathbf{r}_{1}\right)=0 \Longrightarrow \frac{\partial \mathbf{r}^{1}}{\partial(\ldots)} \cdot \mathbf{r}_{1}+\mathbf{r}^{1} \cdot \frac{\partial \mathbf{r}_{1}}{\partial(\ldots)}=0 \Longrightarrow \\
\frac{\partial \mathbf{r}^{1}}{\partial(\ldots)} \cdot \mathbf{r}_{1}=-\mathbf{r}^{1} \cdot \frac{\partial \mathbf{r}_{1}}{\partial(\ldots)} .
\end{gathered}
$$

Now, we can compute the Christoffel symbols directly

$$
\left(\Gamma_{11}^{1}\right)_{\zeta=0}=-\left(\frac{\partial \mathbf{r}_{1}}{\partial \xi} \cdot \mathbf{r}^{1}\right)_{\zeta=0}=-\frac{\partial}{\partial \xi}\left(\left(1-\frac{h_{11}}{a_{11}} \zeta\right) \boldsymbol{\rho}_{\xi}\right)_{\zeta=0} \cdot \boldsymbol{\rho}^{1}=
$$

the contravariant basis vector $\boldsymbol{\rho}^{1}$ is expressed via the covariant one in eqn. (9)

$$
=-\left(\frac{\partial}{\partial \xi}\left(1-\frac{h_{11}}{a_{11}} \zeta\right) \boldsymbol{\rho}_{\xi}+\left(1-\frac{h_{11}}{a_{11}} \zeta\right) \boldsymbol{\rho}_{\xi \xi}\right)_{\zeta=0} \cdot \frac{\boldsymbol{\rho}_{\xi}}{\left(\boldsymbol{\rho}_{\xi} \cdot \boldsymbol{\rho}_{\xi}\right)}=-\frac{\boldsymbol{\rho}_{\xi \xi} \cdot \boldsymbol{\rho}_{\xi}}{\boldsymbol{\rho}_{\xi} \cdot \boldsymbol{\rho}_{\xi}}=-\Gamma .
$$

From eqns. (11) and (12) the identity mit $-\Gamma$ is found.

The last necessary Christoffel symbol is computed analogously

$$
\left(\Gamma_{21}^{1}\right)_{\zeta=0}=-\left(\frac{\partial \mathbf{r}_{1}}{\partial \zeta} \cdot \mathbf{r}^{1}\right)_{\zeta=0}=-\frac{\partial}{\partial \zeta}\left(\left(1-\frac{h_{11}}{a_{11}} \zeta\right) \boldsymbol{\rho}_{\xi}\right)_{\zeta=0} \cdot \boldsymbol{\rho}^{1}=
$$




$$
=\frac{h_{11}}{a_{11}} \boldsymbol{\rho}_{\xi} \cdot \frac{\boldsymbol{\rho}_{\xi}}{\left(\boldsymbol{\rho}_{\xi} \cdot \boldsymbol{\rho}_{\xi}\right)}=\frac{h_{11}}{a_{11}} .
$$

Finally, the tangent component of the full derivative in eqn. (95) gets the following form:

$$
\frac{D_{1} T}{d t}:=\frac{\partial T}{\partial t}+\frac{\partial T}{\partial \xi} \dot{\xi}+T\left(\Gamma_{11}^{1} \dot{\xi}+\Gamma_{21}^{1} \dot{\zeta}\right)_{\zeta=0}=\frac{d T}{d t}-T\left(\frac{\boldsymbol{\rho}_{\xi \xi} \cdot \boldsymbol{\rho}_{\xi}}{\boldsymbol{\rho}_{\xi} \cdot \boldsymbol{\rho}_{\xi}} \dot{\xi}-\frac{h_{11}}{a_{11}} \dot{\zeta}\right) .
$$




\section{References}

[1] Gray A. Modern Differential Geometry of Curves and Surfaces. Boca Raton: CRC Press, 1993.

[2] Harnau M, Konyukhov A, Schweizerhof K. Algorithmic aspects in large deformation contact analysis using "Solid-Shell" elements. Computers and Structures, Available online 21 April 2005.

[3] Hauptmann R, Schweizerhof K. A systematic development of 'solid-shell' element formulations for linear and non-linear analysis employing only displacement degrees of freedom. International Journal for Numerical Methods in Engineering 1998; 42:49-69.

[4] Hauptmann R, Doll S, Harnau M, Schweizerhof K. "Solid-shell" elements with linear and quadratic shape functions at large deformations with nearly incompressible materials. Computers and Structures 2001; 79:1671-1685.

[5] Kikuchi N, Oden JT. Contact Problems in Elasticity: a study of variational inequalities and finite element methods. Philadelphia: SIAM, 1988.

[6] Konyukhov A, Schweizerhof K. Contact formulation via a velocity description allowing efficiency improvements in frictionless contact analysis. Computational Mechanics 2004; 33:165-173.

[7] Konyukhov A, Schweizerhof K. Covariant description for frictional contact problems. Computational Mechanics 2005; 35:190-213.

[8] Krstulovic-Opara L, Wriggers P, Korelc J. A $C^{1}$-continuous formulation for 3D finite deformation frictional contact. Computational Mechanics 2002; 29:27-42.

[9] Laursen TA, Simo JC. A continuum-based finite element formulation for the implicit solution of multibody large deformation frictional contact problems. International Journal for Numerical Methods in Engineering 1993; 35:3451-3485.

[10] Laursen TA. Computational Contact and Impact Mechanics. Fundamentals of Modeling Interfacial Phenomena in Nonlinear Finite Element Analysis. Berlin; Heidelberg; New York: Springer, 2002.

[11] Marsden JE, Hughes TJR. Mathematical Foundations of Elasticity. New York: Dover, 1994. 
[12] McDevitt TW, Laursen TA. A mortar-finite element formulation for frictional contact problems. International Journal for Numerical Methods in Engineering 2000; 48:1525-1547.

[13] Padmanabhan V, Laursen TA. A framework for development of surface smoothing procedures in large deformation frictional contact analysis. Finite Elements in Analysis and Design 2001; 37:173-198.

[14] Parisch H. A consistent tangent stiffness matrix for three-dimensional nonlinear contact analysis. International Journal for Numerical Methods in Engineering 1989; 28:1803-1812.

[15] Parisch H, Lübbing Ch. A formulation of arbitrarily shaped surface elements for three-dimensional large deformation contact with friction. International Journal for Numerical Methods in Engineering 1997; 40:33593383 .

[16] Peric D, Owen DRJ. Computational model for 3-D contact problems with friction based on the penalty method. International Journal for Numerical Methods in Engineering 1992; 35:1289-1309.

[17] Puso MA, Laursen TA. A 3D contact smoothing method using Gregory patches. International Journal For Numerical Methods in Engineering 2002; 54:1161-1194.

[18] Puso MA, Laursen TA. A mortar segment-to-segment contact method for large deformation solid mechanics. Computer Methods in Applied Mechanics and Engineering 2004; 193:601-629.

[19] Stadler M, Holzapfel GA, Korelc J. $C^{n}$ continuous modeling of smooth contact surfaces using NURBS and application to 2D problems. International Journal for Numerical Methods in Engineering 2003; 57:2177-2203.

[20] Stadler M, Holzapfel GA. Subdivision schemes for smooth contact surfaces of arbitrary mesh topology in 3D. International Journal for Numerical Methods in Engineering 2004; 60:1161-1195.

[21] Taylor RL. FEAP - A Finite Element Analysis Program. University of California at Berkeley, Berkeley. Schweizerhof K. and Coworkers, FEAP Mechanik - Karlsruhe. Institut für Mechanik, Universität Karlsruhe, 1987.

[22] Wriggers P, Krstulovic-Opara L, Korelc J. Smooth $C^{1}$-interpolations for two-dimensional frictional contact problems. International Journal for $\mathrm{Nu}$ merical Methods in Engineering 2001; 51:1469-1495. 
[23] Wriggers P, Vu Van, Stein E. Finite element formulation of large deformation impact-contact problems with friction. Computers and Structures 1990; 37:319-331.

[24] Wriggers P. Finite element algorithms for contact problems. Archives of Computational Methods in Engineering 1995; 2(4):1-49.

[25] Wriggers P, Scherf O. Different a posteriori error estimators and indicators for contact problems. Mathematical and Computer Modelling 1998; 28:437447.

[26] Wriggers P. Computational Contact Mechanics. Chichester: Wiley, 2002.

[27] Zavarise G, Wriggers P. A segment-to-segment contact strategy. Mathematical and Computer Modelling 1998; 28:497-515. 